The Journal of Symbolic Logic

Volume 86, Number 3, September 2021

\title{
HOD IN INNER MODELS WITH WOODIN CARDINALS
}

\author{
SANDRA MÜLLER AND GRIGOR SARGSYAN
}

\begin{abstract}
We analyze the hereditarily ordinal definable sets HOD in $M_{n}(x)[g]$ for a Turing cone of reals $x$, where $M_{n}(x)$ is the canonical inner model with $n$ Woodin cardinals build over $x$ and $g$ is generic over $M_{n}(x)$ for the Lévy collapse up to its bottom inaccessible cardinal. We prove that assuming $\boldsymbol{\Pi}_{n+2^{-}}^{1}$ determinacy, for a Turing cone of reals $x, \operatorname{HOD}^{M_{n}(x)[g]}=M_{n}\left(\mathcal{M}_{\infty} \mid \kappa_{\infty}, \Lambda\right)$, where $\mathcal{M}_{\infty}$ is a direct limit of iterates of $M_{n+1}, \delta_{\infty}$ is the least Woodin cardinal in $\mathcal{M}_{\infty}, \kappa_{\infty}$ is the least inaccessible cardinal in $\mathcal{M}_{\infty}$ above $\delta_{\infty}$, and $\Lambda$ is a partial iteration strategy for $\mathcal{M}_{\infty}$. It will also be shown that under the same hypothesis $\mathrm{HOD}^{M_{n}(x)[g]}$ satisfies GCH.
\end{abstract}

\$1. Introduction. An essential question regarding the theory of inner models is the analysis of the class of all hereditarily ordinal definable sets HOD inside various inner models $M$ of the set theoretic universe $V$ under appropriate determinacy hypotheses. Examples for such inner models $M$ are $L(\mathbb{R}), L[x]$, and the canonical proper class $x$-mouse with $n$ Woodin cardinals $M_{n}(x)$, but nowadays also larger models of determinacy $M$ are considered.

One motivation for analyzing the internal structure of these models $\mathrm{HOD}^{M}$ is given by Woodin's results in [5] that under determinacy hypotheses these models contain large cardinals. He showed in [5] for example that assuming $\Delta_{2}^{1}$ determinacy there is a Turing cone of reals $x$ such that $\omega_{2}^{L[x]}$ is a Woodin cardinal in the model $\operatorname{HOD}^{L[x]}$. This result generalizes to higher levels in the projective hierarchy. That means for $n \geq 1$ assuming $\Pi_{n+1}^{1}$ determinacy and $\Pi_{n+2}^{1}$ determinacy there is a cone of reals $x$ such that $\omega_{2}^{M_{n}(x)}$ is a Woodin cardinal in the model $\operatorname{HOD}^{M_{n}(x) \mid \delta_{x}}$, where $M_{n}(x)$ denotes the canonical proper class $x$-mouse with $n$ Woodin cardinals and $\delta_{x}$ is the least Woodin cardinal in $M_{n}(x)$. Moreover, Woodin showed a similar result for $\mathrm{HOD}^{L(\mathbb{R})}$. If we let $\Theta$ denote the supremum of all ordinals $\alpha$ such that there exists a surjection $\pi: \mathbb{R} \rightarrow \alpha$, then assuming $\mathrm{ZF}+\mathrm{AD}$, he showed that $\Theta^{L(\mathbb{R})}$ is a Woodin cardinal in $\mathrm{HOD}^{L(\mathbb{R})}$ (see [5]). The fact that these models of the form $\mathrm{HOD}^{M}$ can have large cardinals as for example Woodin cardinals motivates the question if they are in some sense fine structural as for example the models $L[x], M_{n}(x)$, and $L(\mathbb{R})$ are. A good test question for this is whether these models HOD ${ }^{M}$ satisfy the generalized continuum hypothesis GCH. If it turns out that $\mathrm{HOD}^{M}$ is in fact a

Received January 9, 2018.

2020 Mathematics Subject Classification. 03E45, 03E60, 03E55.

Key words and phrases. HOD, determinacy, inner model theory, large cardinal, Woodin cardinal, mouse.

(C) The Author(s), 2021. Published by Cambridge University Press on behalf of Association for Symbolic Logic. This is an Open Access article, distributed under the terms of the Creative Commons Attribution licence (https://creativecommons.org/licenses/by/4.0/), which permits unrestricted re-use, distribution, and reproduction in any medium, provided the original work is properly cited. 
fine structural model, it would follow that it satisfies the $\mathrm{GCH}$ and even stronger combinatorial principles as for example the $\diamond$ principle.

The first model which was analyzed in this sense was $\operatorname{HOD}^{L(\mathbb{R})}$ under the assumption that every set of reals in $L(\mathbb{R})$ is determined (short: $\mathrm{AD}^{L(\mathbb{R})}$ ). Using purely descriptive set theoretic methods Becker showed in [1] under this hypothesis that $\mathrm{GCH}_{\alpha}$, i.e., $2^{\alpha}=\alpha^{+}$, holds in $\mathrm{HOD}^{L(\mathbb{R})}$ for all $\alpha<\omega_{1}$. Later Steel and Woodin were able to push the analysis of $\operatorname{HOD}^{L(\mathbb{R})}$ forward using more recent advances in inner model theory. In 1993 they first showed independently that the reals in $\operatorname{HOD}^{L(\mathbb{R})}$ are the same as the reals in $M_{\omega}$, the least proper class iterable premouse with $\omega$ Woodin cardinals. Then they showed in Section 4 of [18] that $\operatorname{HOD}^{L(\mathbb{R})}$ in fact agrees with the inner model $N$ up to $\mathcal{P}\left(\omega_{1}\right)$, where $N$ denotes the $\omega_{1}$-th linear iterate of $M_{\omega}$ by its least measure and its images. Building on this, Steel was able to show in [19] that $\operatorname{HOD}^{L(\mathbb{R})}$ agrees with the inner model $\mathcal{M}_{\infty}$ up to $\left(\delta_{1}^{2}\right)^{L(\mathbb{R})}$, where $\mathcal{M}_{\infty}$ is a direct limit of iterates of $M_{\omega}$ and $\left(\delta_{1}^{2}\right)^{L(\mathbb{R})}$ is the supremum of all ordinals $\alpha$ such that there exists a surjection $\pi: \mathbb{R} \rightarrow \alpha$ which is $\Delta_{1}^{L(\mathbb{R})}$ definable. Finally, in 1996 Woodin extended this (see [23]) and showed that in fact $\operatorname{HOD}^{L(\mathbb{R})}=L\left[\mathcal{M}_{\infty}, \Lambda\right]$, where $\Lambda$ is a partial iteration strategy for $\mathcal{M}_{\infty}$. For even larger models of determinacy $M$ the corresponding model $\mathrm{HOD}^{M}$ was first analyzed in [11], where the second author showed that it is fine structural using a layered hierarchy. Models of this form are nowadays called hod mice. A different approach for the fine structure of hod mice called the least branch hierarchy is studied in [22].

The question if $\mathrm{HOD}^{L[x]}$ is a model of $\mathrm{GCH}$ or even a fine structural model for a Turing cone of reals $x$ under a suitable determinacy hypothesis remains open until today. What has been done is the analysis of the model $\operatorname{HOD}^{L[x][G]}$, where $G$ is $\operatorname{Col}\left(\omega,<\kappa_{x}\right)$-generic over $\operatorname{HOD}^{L[x]}$ for the least inaccessible cardinal $\kappa_{x}$ in $L[x]$. Woodin showed in the 1990's (see [23]) that assuming $\Delta_{2}^{1}$ determinacy there is a Turing cone of reals $x$ such that $\operatorname{HOD}^{L[x][G]}=L\left[\mathcal{M}_{\infty}, \Lambda\right]$, where $\mathcal{M}_{\infty}$ is a direct limit of mice (which are iterates of $M_{1}$ ) and $\Lambda$ is a partial iteration strategy for $\mathcal{M}_{\infty}$.

In this article, we analyze HOD in the model $M_{n}(x)[g]$ for any real $x$ of sufficiently high Turing degree under the assumption that every $\boldsymbol{\Pi}_{n+2}^{1}$ set of reals is determined. Here $g$ is $\operatorname{Col}(\omega,<\kappa)$-generic over $M_{n}(x)$, where $\kappa$ denotes the least inaccessible cardinal in $M_{n}(x)$. We first show that the direct limit model $\mathcal{M}_{\infty}$, obtained from iterates of suitable premice, agrees up to its bottom Woodin cardinal $\delta_{\infty}$ with $\operatorname{HOD}^{M_{n}(x)[g]}$. In a second step, we show that the full model $\mathrm{HOD}^{M_{n}(x)[g]}$ is in fact of the form $M_{n}\left(\hat{\mathcal{M}}_{\infty} \mid \kappa_{\infty}, \Lambda\right)$, where $\hat{\mathcal{M}}_{\infty}=M_{n}\left(\mathcal{M}_{\infty} \mid \delta_{\infty}\right), \kappa_{\infty}$ is the least inaccessible cardinal of $\hat{\mathcal{M}}_{\infty}$ above $\delta_{\infty}$, and $\Lambda$ is a partial iteration strategy for $\mathcal{M}_{\infty}$. Here and below $M_{n}\left(\hat{\mathcal{M}}_{\infty} \mid \kappa_{\infty}, \Lambda\right)$ denotes the canonical fine structural model with $n$ Woodin cardinals build over the coarse objects $\hat{\mathcal{M}}_{\infty} \mid \kappa_{\infty}$ and $\Lambda$. Our proof in fact shows that $\operatorname{HOD}^{M_{n}(x)[g]}$ is a model of $\mathrm{GCH}, \diamond$, and other combinatorial principles which are consequences of fine structure.

In the statement of the following main theorem and in fact everywhere in this article whenever we write $\mathrm{HOD}^{M}$ for some premouse $M$ we mean $\operatorname{HOD}^{\lfloor M\rfloor}$, where $\lfloor M\rfloor$ denotes the universe of the model $M$. In particular, we do not allow the extender sequence of $M$ as a parameter in the definition of HOD. It will be clear from the 
context if we consider the model $M$ or the universe $\lfloor M\rfloor$ of $M$, therefore we decided for the sake of readability to not distinguish the notation for these two objects.

The main result of this paper is the following theorem.

THeOREM 1.1. Let $n<\omega$ and assume $\mathbf{\Pi}_{n+2}^{1}$-determinacy. Then for a Turing cone of reals $x$,

$$
\operatorname{HOD}^{M_{n}(x)[g]}=M_{n}\left(\hat{\mathcal{M}}_{\infty} \mid \kappa_{\infty}, \Lambda\right),
$$

where $g$ is $\operatorname{Col}(\omega,<\kappa)$-generic over $M_{n}(x), \kappa$ denotes the least inaccessible cardinal in $M_{n}(x), \hat{\mathcal{M}}_{\infty}$ is a direct limit of iterates of $M_{n+1}, \delta_{\infty}$ is the least Woodin cardinal in $\hat{\mathcal{M}}_{\infty}, \kappa_{\infty}$ is the least inaccessible cardinal of $\hat{\mathcal{M}}_{\infty}$ above $\delta_{\infty}$, and $\Lambda$ is a partial iteration strategy for $\mathcal{M}_{\infty}$.

Our proof in fact shows the following corollary.

COROllary 1.2. Assume $\mathbf{\Pi}_{n+2}^{1}$-determinacy. Then for a Turing cone of reals $x$,

$$
\mathrm{HOD}^{M_{n}(x)[g]} \vDash \mathrm{GCH},
$$

where $g$ is $\operatorname{Col}(\omega,<\kappa)$-generic over $M_{n}(x)$ and $\kappa$ denotes the least inaccessible cardinal in $M_{n}(x)$.

REMARK. In fact the full strength of $\boldsymbol{\Pi}_{n+2}^{1}$-determinacy is not needed for these results. It suffices to assume that $M_{n}^{\#}(x)$ exists and is $\omega_{1}$-iterable for all reals $x$ (or equivalently $\Pi_{n+1}^{1}$-determinacy, see [8] and [10]) and that $M_{n+1}^{\#}$ exists and is $\omega_{1}$-iterable. This is all we will use in the proof.

Finally, we summarize some open questions related to these results. The following question already appears in [23].

Question 1. Assume $\Delta_{2}^{1}$ determinacy. Is $\mathrm{HOD}^{L[x]}$ for a cone of reals $x$ a fine structural model?

QUestion 2. Assume $\mathbf{\Pi}_{n+2}^{1}$ determinacy. Is $\operatorname{HOD}^{M_{n}(x)}$ for a cone of reals $x$ a fine structural model?

This article is structured as follows. In Section 2 we recall some preliminaries and fix the basic notation. In Section 3 we recall the relevant notions from [12] and define the direct limit system converging to $\mathcal{M}_{\infty}$, before we compute $\operatorname{HOD}^{M_{n}(x)[g]}$ up to its Woodin cardinal in Section 4. In Section 5 we then show how this can be used to compute the full model $\mathrm{HOD}^{M_{n}(x)[g]}$, i.e., we finish the proof of Theorem 1.1.

§2. Preliminaries and notation. Whenever we say reals we mean elements of the Baire space ${ }^{\omega} \omega$. We also write $\mathbb{R}$ for ${ }^{\omega} \omega$. HOD denotes the class of all hereditarily ordinal definable sets. Moreover $\mathrm{HOD}_{x}$ for any $x \in{ }^{\omega} \omega$ denotes the class of all sets which are hereditarily ordinal definable over $\{x\} .{ }^{1}$ That means we let $A \in \mathrm{OD}_{x}$ iff there is a formula $\varphi$ such that $A=\left\{v \mid \varphi\left(v, \alpha_{1}, \ldots, \alpha_{n}, x\right)\right\}$ for some ordinals $\alpha_{1}, \ldots, \alpha_{n}$. Then $A \in \mathrm{HOD}_{x}$ iff $\mathrm{TC}(\{A\}) \subset \mathrm{OD}_{x}$, where $\mathrm{TC}(\{A\})$ denotes the transitive closure of the set $\{A\}$.

\footnotetext{
${ }^{1}$ In the literature this is sometimes also called $\operatorname{HOD}_{\{x\}}$.
} 
We use the notions of premice and iterability from [21, Sections 1-4] and assume that the reader is familiar with the basic concepts defined there. In most cases we will demand $\left(\omega, \omega_{1}, \omega_{1}\right)$-iterability in the sense of Definition 4.4 in [21] for our mice, but in other cases or if it is not clear from the context we will state the precise amount of iterability. We say a cutpoint of a premouse $\mathcal{M}$ is an infinite ordinal $\gamma$ such that there is no extender $E$ on the $\mathcal{M}$-sequence with $\operatorname{crit}(E) \leq \gamma \leq \operatorname{lh}(E){ }^{2}$

For some ZFC model $M$ and some real $x \in M$ we write $L[E](x)^{M}$ for the result of a fully backgrounded extender construction above $x$ inside $M$ in the sense of [7], with the minimality condition relaxed to $\omega$-small premice. Moreover, we let for a premouse $\mathcal{M}$ with $\mathcal{M} \vDash \mathrm{ZFC}$, a cardinal cutpoint $\eta$ of $\mathcal{M}$, and a premouse $\mathcal{N}$ of height $\eta$ such that $\mathcal{N} \in \mathcal{P}(\mathcal{M} \mid \eta) \cap \mathcal{M} \mid(\eta+\omega), \mathcal{P}^{\mathcal{M}}(\mathcal{N})$ denote the result of a $\mathcal{P}$-construction over $\mathcal{N}$ inside the model $\mathcal{M}$ in the sense of [15] or [12, Proposition 2.3 and Definition 2.4].

For $x \in{ }^{\omega} \omega$ and $n \leq \omega$ we let $M_{n}^{\#}(x)$, if it exists, denote a countable, sound, $\omega_{1}$-iterable $x$-premouse which is not $n$-small but all of whose proper initial segments are $n$-small. In fact, $\omega_{1}$-iterability suffices to show that such an $M_{n}^{\#}(x)$ is unique. If $M_{n}^{\#}(x)$ exists, we let $M_{n}(x)$ be the proper class premouse obtained by iterating the top extender of $M_{n}^{\#}(x)$ out of the universe.

§3. The direct limit system. To show that $\operatorname{HOD}^{M_{n}(x)[g]}$ is a fine structural inner model, we will use an extension of the direct limit system introduced in [12]. For the reader's convenience we will first recall the relevant definitions and results from [12], obtaining a direct limit system which is definable in $M_{n}(x)$. We use the chance to correct some minor errors in the presentation of that direct limit system in [12]. Then we discuss the changes we need to make to obtain a direct limit system definable in $M_{n}(x)[g]$. Another application of a similar but slightly different direct limit system as in [12] can be found in [13].

Fix an arbitrary natural number $n$. Throughout the rest of this article we will assume that $M_{n+1}^{\#}$ exists and is $\left(\omega, \omega_{1}, \omega_{1}\right)$-iterable and fix a real $x$ that codes $M_{n+1}^{\#}$. This implies $\boldsymbol{\Pi}_{n+1}^{1}$ determinacy or equivalently that $M_{n}^{\#}(z)$ exists and is $\left(\omega, \omega_{1}, \omega_{1}\right)$ iterable for all reals $z$ (see [9] and [8] for a proof of this equivalence due to Neeman and Woodin). Finally, we fix a $\operatorname{Col}(\omega,<\kappa)$-generic $g$ over $M_{n}(x)$, where $\kappa$ is the least inaccessible cardinal in $M_{n}(x)$.

The first direct limit system. We first recall the definition of a lower part premouse.

Definition 3.1. Let $a$ be a countable, transitive, self-wellordered ${ }^{3}$ set. Then we define the lower part model $L p^{n}(a)$ as the model theoretic union of all countable $a$-premice $\mathcal{M}$ with $\rho_{\omega}(\mathcal{M})=a$ which are $n$-small, sound, and $\left(\omega, \omega_{1}, \omega_{1}\right)$-iterable.

If $\mathcal{N}$ is a countable premouse, we also use $L p^{n}(\mathcal{N})$ to denote the premouse extending $\mathcal{N}$ which is defined similarly as the model theoretic union of premice $\mathcal{M} \unrhd \mathcal{N}$ with $\rho_{\omega}(\mathcal{M}) \leq \mathcal{N} \cap$ Ord which have $\mathcal{N} \cap$ Ord as a cutpoint, are $n$-small above $\mathcal{N} \cap$ Ord, sound above $\mathcal{N} \cap$ Ord, and $\left(\omega, \omega_{1}, \omega_{1}\right)$-iterable above $\mathcal{N} \cap$ Ord. In

\footnotetext{
${ }^{2}$ Such a cutpoint $\gamma$ is often also called a strong cutpoint.

${ }^{3}$ We say a transitive set $a$ is self-wellordered iff $a$ is wellordered in $L_{\omega}[a]$.
} 
case $\mathcal{M} \unrhd \mathcal{N}$ has a partial measure $\mu$ with critical point $\mathcal{N} \cap$ Ord, we replace $\mathcal{M}$ by the corresponding translated premouse in which $\mathcal{N} \cap$ Ord is a cutpoint as in [20, Remark 12.7] in order to include it in $L p^{n}(\mathcal{N})$ as well.

Definition 3.2. A countable premouse $\mathcal{N}$ is $n$-suitable iff there is an ordinal $\delta$ such that

(1) $\mathcal{N} \vDash$ "ZFC - Replacement" and $\mathcal{N} \cap \operatorname{Ord}=\sup _{i<\omega}\left(\delta^{+i}\right)^{\mathcal{N}}$,

(2) $\mathcal{N} \vDash$ " $\delta$ is a Woodin cardinal",

(3) $\mathcal{N}$ is $(n+1)$-small,

(4) for every cutpoint $\gamma<\delta$ of $\mathcal{N}, \gamma$ is not Woodin in $L p^{n}(\mathcal{N} \mid \gamma)$,

(5) $\mathcal{N} \mid\left(\delta^{+(i+1)}\right)^{\mathcal{N}}=L p^{n}\left(\mathcal{N} \mid\left(\delta^{+i}\right)^{\mathcal{N}}\right)$ for all $i<\omega$, and

(6) for all $\eta<\delta, \mathcal{N} \vDash " \mathcal{N} \mid \delta$ is $(\omega, \eta, \eta)$-iterable."

If $\mathcal{N}$ is an $n$-suitable premouse we denote the ordinal $\delta$ from Definition 3.2 by $\delta^{\mathcal{N}}$. Moreover, we write $\hat{\mathcal{N}}=M_{n}\left(\mathcal{N} \mid \delta^{\mathcal{N}}\right)$ for any $n$-suitable premouse $\mathcal{N}$. Then $\mathcal{N}=\hat{\mathcal{N}} \mid\left(\left(\delta^{\mathcal{N}}\right)^{+\omega}\right)^{\hat{\mathcal{N}}}$ for every $n$-suitable premouse $\mathcal{N}$ by well-known properties of the lower part model $L p^{n}$. We now give some definitions indicating how $n$-suitable premice can be iterated.

DeFINITION 3.3. Let $\mathcal{N}$ be an arbitrary premouse and let $\mathcal{T}$ be an iteration tree on $\mathcal{N}$ of limit length.

(1) We say a premouse $\mathcal{Q}=\mathcal{Q}(\mathcal{T})$ is a $\mathcal{Q}$-structure for $\mathcal{T}$ iff $\mathcal{M}(\mathcal{T}) \unlhd \mathcal{Q}$, $\mathcal{Q}$ is sound above $\delta(\mathcal{T}), \delta(\mathcal{T})$ is a cutpoint of $\mathcal{Q}, \mathcal{Q}$ is $\left(\omega, \omega_{1}, \omega_{1}\right)$-iterable above $\delta(\mathcal{T})$, and if $\mathcal{Q} \neq \mathcal{M}(\mathcal{T})$

$$
\mathcal{Q} \vDash " \delta(\mathcal{T}) \text { is a Woodin cardinal, } "
$$

and

(i) over $\mathcal{Q}$ there exists an $r \Sigma_{n}$-definable set $A \subset \delta(\mathcal{T})$ such that there is no $\kappa<\delta(\mathcal{T})$ such that $\kappa$ is strong up to $\delta(\mathcal{T})$ with respect to $A$ as being witnessed by extenders on the sequence of $\mathcal{Q}$ for some $n<\omega$, or

(ii) $\rho_{n}(\mathcal{Q})<\delta(\mathcal{T})$ for some $n<\omega$.

(2) Let $b$ be a cofinal well-founded branch through $\mathcal{T}$. Then we say a premouse $\mathcal{Q}=\mathcal{Q}(b, \mathcal{T})$ is a $\mathcal{Q}$-structure for $b$ in $\mathcal{T}$ iff $\mathcal{Q}=\mathcal{M}_{b}^{\mathcal{T}} \mid \gamma$, where $\gamma \leq \mathcal{M}_{b}^{\mathcal{T}} \cap$ Ord is the least ordinal such that either

$$
\gamma<\mathcal{M}_{b}^{\mathcal{T}} \cap \text { Ord and } \mathcal{M}_{b}^{\mathcal{T}} \mid(\gamma+1) \vDash " \delta(\mathcal{T}) \text { is not Woodin," }
$$

or

$$
\gamma=\mathcal{M}_{b}^{\mathcal{T}} \cap \text { Ord and } \rho_{n}\left(\mathcal{M}_{b}^{\mathcal{T}}\right)<\delta(\mathcal{T})
$$

for some $n<\omega$ or over $\mathcal{M}_{b}^{\mathcal{T}}$ there exists an $r \Sigma_{n}$-definable set $A \subset \delta(\mathcal{T})$ such that there is no $\kappa<\delta(\mathcal{T})$ such that $\kappa$ is strong up to $\delta(\mathcal{T})$ with respect to $A$ as being witnessed by extenders on the sequence of $\mathcal{M}_{b}^{\mathcal{T}}$ for some $n<\omega$.

If no such ordinal $\gamma \leq \mathcal{M}_{b}^{\mathcal{T}} \cap$ Ord exists, we let $\mathcal{Q}(b, \mathcal{T})$ be undefined. 
REMARK. The premouse $M_{n+1} \mid\left(\delta_{0}^{+\omega}\right)^{M_{n+1}}$ is $n$-suitable, where $\delta_{0}$ is the least Woodin cardinal in $M_{n+1}$. We denote this premouse by $M_{n+1}^{-}$and write $\Sigma_{M_{n+1}^{-}}$ for its canonical iteration strategy induced by the usual iteration strategy $\Sigma_{M_{n+1}}$ for $M_{n+1}$ for countable stacks of normal trees without drops on the main branches.

Our goal is to approximate the iteration strategy $\Sigma_{M_{n+1}^{-}}$inside $\operatorname{HOD}^{M_{n}(x)[g]}$. Analogous to [17, Definition 5.32] we define the following requirement, which will be used in Definition 3.6 to make the proof of Lemmas 3.8 and 3.9 work.

Definition 3.4. Let $\mathcal{N}$ be an $n$-suitable premouse and let $\mathcal{T}$ be a normal iteration tree on $\mathcal{N}$ of length $<\omega_{1}^{V}$. Then we say that $\mathcal{T}$ is suitability strict iff for all $\alpha<\operatorname{lh}(\mathcal{T})$,

(i) if $[0, \alpha]_{T}$ does not drop then $\mathcal{M}_{\alpha}^{\mathcal{T}}$ is $n$-suitable, and

(ii) if $[0, \alpha]_{T}$ drops then no $\mathcal{R} \unlhd \mathcal{M}_{\alpha}^{\mathcal{T}}$ is $n$-suitable.

DeFinition 3.5. Let $\mathcal{N}$ be an $n$-suitable premouse and let $\mathcal{T}$ be a normal iteration tree on $\mathcal{N}$ of length $<\omega_{1}^{V}$.

(1) $\mathcal{T}$ is correctly guided iff for every limit ordinal $\lambda<\operatorname{lh}(\mathcal{T})$, if $b$ is the branch choosen for $\mathcal{T} \uparrow \lambda$ in $\mathcal{T}$, then $\mathcal{Q}(b, \mathcal{T} \uparrow \lambda)$ exists and $\mathcal{Q}(b, \mathcal{T} \uparrow \lambda) \unlhd$ $M_{n}(\mathcal{M}(\mathcal{T}\lceil\lambda))$

(2) $\mathcal{T}$ is short iff $\mathcal{T}$ is correctly guided and in case $\mathcal{T}$ has limit length $\mathcal{Q}(\mathcal{T})$ exists and $\mathcal{Q}(\mathcal{T}) \unlhd M_{n}(\mathcal{M}(\mathcal{T}))$.

(3) $\mathcal{T}$ is maximal iff $\mathcal{T}$ is correctly guided and not short.

Definition 3.6. Let $\mathcal{N}$ be an $n$-suitable premouse. We say $\mathcal{N}$ is short tree iterable iff whenever $\mathcal{T}$ is a short tree on $\mathcal{N}$,

(i) $\mathcal{T}$ is suitability strict,

(ii) if $\mathcal{T}$ has a last model, then every putative ${ }^{4}$ iteration tree $\mathcal{U}$ extending $\mathcal{T}$ such that $\operatorname{lh}(\mathcal{U})=\operatorname{lh}(\mathcal{T})+1$ has a well-founded last model, and

(iii) if $\mathcal{T}$ has limit length, then there exists a cofinal well-founded branch $b$ through $\mathcal{T}$ such that $\mathcal{Q}(b, \mathcal{T})=\mathcal{Q}(\mathcal{T})$.

This can be generalized to stacks of correctly guided normal trees.

Definition 3.7. Let $\mathcal{N}$ be an $n$-suitable premouse and $m<\omega$. Then we say $\left(\mathcal{T}_{i}, \mathcal{N}_{i} \mid i \leq m\right)$ is a correctly guided finite stack on $\mathcal{N}$ iff

(i) $\mathcal{N}_{0}=\mathcal{N}$

(ii) $\mathcal{N}_{i}$ is $n$-suitable and $\mathcal{T}_{i}$ is a correctly guided normal iteration tree on $\mathcal{N}_{i}$ which acts below $\delta^{\mathcal{N}_{i}}$ for all $i \leq m$, and

(iii) for every $i<m$ either $\mathcal{T}_{i}$ has a last model which is equal to $\mathcal{N}_{i+1}$ and the iteration embedding $i \mathcal{T}_{i}: \mathcal{N}_{i} \rightarrow \mathcal{N}_{i+1}$ exists or $\mathcal{T}_{i}$ is maximal and $\mathcal{N}_{i+1}=$ $M_{n}\left(\mathcal{M}\left(\mathcal{T}_{i}\right)\right) \mid\left(\delta\left(\mathcal{T}_{i}\right)^{+\omega}\right)^{M_{n}\left(\mathcal{M}\left(\mathcal{T}_{i}\right)\right)}$.

Moreover, we say that $\mathcal{M}$ is the last model of $\left(\mathcal{T}_{i}, \mathcal{N}_{i} \mid i \leq m\right)$ iff either

(i) $\mathcal{T}_{m}$ has a last model which is equal to $\mathcal{M}$ and the iteration embedding $i^{\mathcal{T}_{m}}$ : $\mathcal{N}_{m} \rightarrow \mathcal{M}$ exists

\footnotetext{
${ }^{4} \mathrm{An}$ iteration tree $\mathcal{U}$ is a putative iteration tree if $\mathcal{U}$ satisfies all properties of an iteration tree, but in case $\mathcal{U}$ has a last model we allow this last model to be ill-founded.
} 
(ii) $\mathcal{T}_{m}$ is of limit length and short and there is a non-dropping cofinal wellfounded branch $b$ through $\mathcal{T}_{m}$ such that $\mathcal{Q}(b, \mathcal{T})$ exists, $\mathcal{T}_{m} \frown b$ is correctly guided, and $\mathcal{M}=\mathcal{M}_{b}^{\mathcal{T}}$, or

(iii) $\mathcal{T}_{m}$ is maximal and $\mathcal{M}=M_{n}\left(\mathcal{M}\left(\mathcal{T}_{m}\right)\right) \mid\left(\delta\left(\mathcal{T}_{m}\right)^{+\omega}\right)^{M_{n}\left(\mathcal{M}\left(\mathcal{T}_{m}\right)\right)}$.

Finally, we say that $\mathcal{M}$ is a correct iterate of $\mathcal{N}$ iff there is a correctly guided finite stack on $\mathcal{N}$ with last model $\mathcal{M}$. In case there is a correctly guided finite stack on $\mathcal{N}$ with last model $\mathcal{M}$ of length 1, i.e., such that $m=0$, we say that $\mathcal{M}$ is a pseudo-normal iterate (or just pseudo-iterate) of $\mathcal{N}$.

Analogous to Theorem 3.14 in [23] we also have a version of the comparison lemma for short tree iterable premice and pseudo-normal iterates.

Lemma 3.8 (Pseudo-comparison lemma). Let $\mathcal{N}$ and $\mathcal{M}$ be $n$-suitable premice which are short tree iterable. Then there is a common pseudo-normal iterate $\mathcal{R} \in M_{n}(y)$ such that $\delta^{\mathcal{R}} \leq \omega_{1}^{M_{n}(y)}$, where $y$ is a real coding $\mathcal{N}$ and $\mathcal{M}$.

The proof of Lemma 3.8 is similar to the proof of Theorem 3.14 in [23], so we omit it. Similarly, we have an analogue to the pseudo-genericity iteration (see Theorem 3.16 in [23]).

LEMMA 3.9 (Pseudo-genericity iterations). Let $\mathcal{N}$ be an $n$-suitable premouse which is short tree iterable and let $z$ be a real. Then there is a pseudo-normal iterate $\mathcal{R}$ of $\mathcal{N}$ in $M_{n}(y, z)$ such that $z$ is $\mathbb{B}^{\mathcal{R}}$-generic over $\mathcal{R}$ and $\delta^{\mathcal{R}} \leq \omega_{1}^{M_{n}(y, z)}$, where $y$ is a real coding $\mathcal{N}$ and $\mathbb{B}^{\mathcal{R}}$ denotes Woodin's extender algebra inside $\mathcal{R}$.

For the definition of the direct limit system converging to HOD we need the notion of $s$-iterability. To define this, we first introduce some notation. For an $n$-suitable premouse $\mathcal{N}$, a finite sequence of ordinals $s$, and some $k<\omega$ let

$$
\begin{gathered}
T_{s, k}^{\mathcal{N}}=\left\{(t,\ulcorner\phi\urcorner) \in\left[\left(\left(\delta^{\mathcal{N}}\right)^{+k}\right)^{\mathcal{N}}\right]^{<\omega} \times \omega \mid \phi \text { is a } \Sigma_{1}\right. \text {-formula and } \\
\left.M_{n}\left(\mathcal{N} \mid \delta^{\mathcal{N}}\right) \vDash \phi[t, s]\right\},
\end{gathered}
$$

where $\ulcorner\phi\urcorner$ denotes the Gödel number of $\phi$. Let $H u l l_{1}^{\mathcal{N}}$ denote an uncollapsed $\Sigma_{1}$ hull in $\mathcal{N}$. Then we let

$$
\gamma_{s}^{\mathcal{N}}=\sup \left(H u l l_{1}^{\mathcal{N}}\left(\left\{T_{s, k}^{\mathcal{N}} \mid k<\omega\right\}\right) \cap \delta^{\mathcal{N}}\right)
$$

and

$$
H_{s}^{\mathcal{N}}=\operatorname{Hull}_{1}^{\mathcal{N}}\left(\gamma_{s}^{\mathcal{N}} \cup\left\{T_{s, k}^{\mathcal{N}} \mid k<\omega\right\}\right) .
$$

Then $\gamma_{s}^{\mathcal{N}}=H_{s}^{\mathcal{N}} \cap \delta^{\mathcal{N}}$. For $s_{m}=\left(u_{1}, \ldots, u_{m}\right)$ the sequence of the first $m$ uniform indiscernibles, we write $\gamma_{m}^{\mathcal{N}}=\gamma_{s_{m}}^{\mathcal{N}}$ and $H_{m}^{\mathcal{N}}=H_{s_{m}}^{\mathcal{N}}$. Then we have that $\sup _{m \in \omega} \gamma_{m}^{\mathcal{N}}=$ $\delta^{\mathcal{N}}$ (see Lemma 5.3 in [12]).

Definition 3.10. Let $\mathcal{N}$ be an $n$-suitable premouse and $s$ a finite sequence of ordinals. Then $\mathcal{N}$ is $s$-iterable iff every correct iterate of $\mathcal{N}$ is short tree iterable and for every correctly guided finite stack $\left(\mathcal{T}_{i}, \mathcal{N}_{i} \mid i \leq m\right)$ on $\mathcal{N}$ with last model $\mathcal{M}$ there is a sequence of non-dropping branches $\left(b_{i} \mid i \leq m\right)$ and a sequence of embeddings $\left(\pi_{i} \mid i \leq m\right)$ such that

(i) if $\mathcal{T}_{i}$ has successor length $\alpha+1$, then $b_{i}=[0, \alpha]_{T_{i}}$ and $\pi_{i}=i_{0, \alpha}^{\mathcal{T}_{i}}$ is the corresponding iteration embedding for $i \leq m$, 
(ii) if $\mathcal{T}_{m}$ is short, then $b_{m}$ is the unique cofinal well-founded branch through $\mathcal{T}_{m}$ such that $\mathcal{Q}\left(b_{m}, \mathcal{T}_{m}\right)$ exists and $\mathcal{T}_{m}{ }^{\frown} b_{m}$ is correctly guided and $\pi_{m}=i_{b_{m}}^{\mathcal{T}_{m}}$ is the corresponding iteration embedding,

(iii) if $\mathcal{T}_{i}$ is maximal, then $b_{i}$ is a cofinal well-founded branch through $\mathcal{T}_{i}$ such that $\mathcal{M}_{b_{i}}^{\mathcal{T}_{i}}=\mathcal{N}_{i+1}$ if $i<m$ or $\mathcal{M}_{b_{i}}^{\mathcal{T}_{i}}=\mathcal{M}$ if $i=m$, and $\pi_{i}=i_{b_{i}}^{\mathcal{T}_{i}}$ is the corresponding iteration embedding for $i \leq m$, and

(iv) if we let $\pi=\pi_{m} \circ \pi_{m-1} \circ \cdots \circ \pi_{0}$ then for every $k<\omega$,

$$
\pi\left(T_{s, k}^{\mathcal{N}}\right)=T_{s, k}^{\mathcal{M}} \text {. }
$$

In this case we say that the sequence $\vec{b}=\left(b_{i} \mid i \leq m\right)$ witnesses $s$-iterability for $\overrightarrow{\mathcal{T}}=\left(\mathcal{T}_{i}, \mathcal{N}_{i} \mid i \leq m\right)$ or that $\vec{b}$ is an $s$-iterability branch for $\overrightarrow{\mathcal{T}}$ and we write $\pi_{\overrightarrow{\mathcal{T}}, \vec{b}}=\pi$.

Now for every two $s$-iterability branches for $\overrightarrow{\mathcal{T}}$ on $\mathcal{N}$ their corresponding iteration embeddings agree on $H_{s}^{\mathcal{N}}$.

Lemma 3.11 (Uniqueness of $s$-iterability embeddings, Lemma 5.5 in [12]). Let $\mathcal{N}$ be an $n$-suitable premouse, $s$ a finite sequence of ordinals, and $\overrightarrow{\mathcal{T}}$ a correctly guided finite stack on $\mathcal{N}$. Moreover let $\vec{b}$ and $\vec{c}$ be s-iterability branches for $\overrightarrow{\mathcal{T}}$. Then

$$
\pi_{\overrightarrow{\mathcal{T}}, \vec{b}} \uparrow H_{s}^{\mathcal{N}}=\pi_{\overrightarrow{\mathcal{T}}, \vec{c}} \uparrow H_{s}^{\mathcal{N}} .
$$

The uniqueness of $s$-iterability embeddings yields that for every $n$-suitable, $s$ iterable $\mathcal{N}$, every correctly guided finite stack $\overrightarrow{\mathcal{T}}$ on $\mathcal{N}$ and every $s$-iterability branch $\vec{b}$ for $\overrightarrow{\mathcal{T}}$, the embedding $\pi_{\overrightarrow{\mathcal{T}}, \vec{b}}\left\lceil H_{s}^{\mathcal{N}}\right.$ is independent of the choice of $\vec{b}$, but it might still depend on $\overrightarrow{\mathcal{T}}$. This motivates the following definition.

Definition 3.12. Let $\mathcal{N}$ be an $n$-suitable premouse and $s$ a finite sequence of ordinals. Then $\mathcal{N}$ is strongly $s$-iterable iff for every correct iterate $\mathcal{R}$ of $\mathcal{N}, \mathcal{R}$ is $s$-iterable and for every two correctly guided finite stacks $\overrightarrow{\mathcal{T}}$ and $\overrightarrow{\mathcal{U}}$ on $\mathcal{R}$ with common last model $\mathcal{M}$ and $s$-iterability witnesses $\vec{b}$ and $\vec{c}$ for $\overrightarrow{\mathcal{T}}$ and $\overrightarrow{\mathcal{U}}$ respectively, we have that

$$
\pi_{\overrightarrow{\mathcal{T}}, \vec{b}}\left\lceil H_{s}^{\mathcal{R}}=\pi_{\overrightarrow{\mathcal{U}}, \vec{c}} \uparrow H_{s}^{\mathcal{R}} .\right.
$$

A so-called bad sequence argument shows the following lemma, which yields the existence of strongly $s$-iterable premice.

Lemma 3.13 (Lemma 5.9 in [12]). For every finite sequence of ordinals $s$ and any short tree iterable $n$-suitable premouse $\mathcal{N}$ there is a pseudo-normal iterate $\mathcal{M}$ of $\mathcal{N}$ such that $\mathcal{M}$ is strongly s-iterable.

If $\mathcal{N}$ is strongly $s$-iterable and $\overrightarrow{\mathcal{T}}$ is a correctly guided finite stack on $\mathcal{N}$ with last model $\mathcal{M}$, let $\pi_{\mathcal{N}, \mathcal{M}, s}: H_{s}^{\mathcal{N}} \rightarrow H_{s}^{\mathcal{M}}$ denote the embedding given by any $s$-iterability branch $\vec{b}$ for $\overrightarrow{\mathcal{T}}$. As $\mathcal{N}$ is strongly $s$-iterable, the embedding $\pi_{\mathcal{N}, \mathcal{M}, s}$ does not depend on the choice of $\overrightarrow{\mathcal{T}}$ and $\vec{b}$.

Recall that we write $M_{n+1}^{-}=M_{n+1} \mid\left(\delta_{0}^{+\omega}\right)^{M_{n+1}}$, where $\delta_{0}$ is the least Woodin cardinal in $M_{n+1}$, and $\Sigma_{M_{n+1}^{-}}$for the canonical iteration strategy for $M_{n+1}^{-}$induced by $\Sigma_{M_{n+1}}$. Moreover, recall that for $m<\omega$, we write $s_{m}$ for the set of the first $m$ uniform indiscernibles. Then $M_{n+1}^{-}$is $n$-suitable and strongly $s_{m}$-iterable for every $m$. Moreover, if $\overrightarrow{\mathcal{T}}$ is a correctly guided finite stack on $M_{n+1}^{-}$with last model $\mathcal{M}$, 
then $\pi_{M_{n+1}^{-}, \mathcal{M}, s_{m}}$ agrees with the iteration embedding according to $\Sigma_{M_{n+1}^{-}}$on $H_{S_{m}}^{M_{n+1}^{-}}$. The first direct limit system we define will consist of iterates of $M_{n+1}^{-}$.

DeFinITION 3.14. Let

$$
\begin{gathered}
\tilde{\mathcal{F}}^{+}=\left\{\mathcal{N} \mid \mathcal{N} \text { is a non-dropping iterate of } M_{n+1}^{-} \text {via } \Sigma_{M_{n+1}^{-}}\right. \\
\text {by a finite stack of countable trees }\}
\end{gathered}
$$

and for $\mathcal{N}, \mathcal{M} \in \tilde{\mathcal{F}}^{+}$let $\mathcal{N} \leq^{+} \mathcal{M}$ iff $\mathcal{M}$ is an iterate of $\mathcal{N}$ via the tail strategy $\Sigma_{\mathcal{N}}$ as witnessed by some finite stack of countable iteration trees. Then we let $\tilde{\mathcal{M}}_{\infty}^{+}$be the direct limit of $\left(\tilde{\mathcal{F}}^{+}, \leq^{+}\right)$under the iteration maps.

REMARK. The prewellordering $\leq_{\tilde{\tilde{F}}}^{+}$on $\tilde{\mathcal{F}}^{+}$is directed and the direct limit $\tilde{\mathcal{M}}_{\infty}^{+}$is well-founded as the limit system $\left(\tilde{\tilde{\mathcal{F}}}^{+}, \leq^{+}\right)$only consists of iterates of $M_{n+1}^{-}$via the canonical iteration strategy $\Sigma_{M_{n+1}^{-}}$.

Since $\tilde{\mathcal{F}}^{+}$is not definable enough for our purposes, we now introduce another direct limit system which has the same direct limit $\tilde{\mathcal{M}}_{\infty}^{+}$.

DEFINITION 3.15. Let

$$
\tilde{\mathcal{I}}=\left\{(\mathcal{N}, s) \mid \mathcal{N} \text { is } n \text {-suitable, } s \in[\text { Ord }]^{<\omega} \text {, and } \mathcal{N} \text { is strongly } s \text {-iterable }\right\}
$$

and

$$
\tilde{\mathcal{F}}=\left\{H_{s}^{\mathcal{N}} \mid(\mathcal{N}, s) \in \tilde{\mathcal{I}}\right\} .
$$

For $(\mathcal{N}, s),(\mathcal{M}, t) \in \tilde{\mathcal{I}}$ we let $(\mathcal{N}, s) \leq_{\tilde{\mathcal{I}}}(\mathcal{M}, t)$ iff there is a correctly guided finite stack on $\mathcal{N}$ with last model $\mathcal{M}$ and $s \subseteq t$. In this case we let $\pi_{(\mathcal{N}, s),(\mathcal{M}, t)}: H_{s}^{\mathcal{N}} \rightarrow H_{t}^{\mathcal{M}}$ denote the canonical corresponding embedding.

REMARK. The prewellordering $\leq_{\tilde{\mathcal{I}}}$ on $\tilde{\mathcal{I}}$ is directed: Let $(\mathcal{N}, s),(\mathcal{M}, t) \in \tilde{\mathcal{I}}$. By Lemma 3.13 there exists an $n$-suitable premouse $\mathcal{R}$ which is strongly $(s \cup t)$-iterable. Let $\mathcal{S}$ be the result of simultaneously comparing $\mathcal{N}, \mathcal{M}$ and $\mathcal{R}$ in the sense of Lemma 3.8. Then $(\mathcal{S}, s \cup t) \in \tilde{\mathcal{I}},(\mathcal{N}, s) \leq_{\tilde{\mathcal{I}}}(\mathcal{S}, s \cup t)$, and $(\mathcal{M}, t) \leq_{\tilde{\mathcal{I}}}(\mathcal{S}, s \cup t)$, as desired.

Definition 3.16. Let $\tilde{\mathcal{M}}_{\infty}$ be the direct limit of $\left(\tilde{\mathcal{F}}, \leq_{\tilde{\mathcal{I}}}\right)$ under the embeddings $\pi_{(\mathcal{N}, s),(\mathcal{M}, t)}$. For $(\mathcal{N}, s) \in \tilde{\mathcal{I}}$ let $\pi_{(\mathcal{N}, s), \infty}: H_{s}^{\mathcal{N}} \rightarrow \tilde{\mathcal{M}}_{\infty}$ denote the corresponding direct limit embedding.

The fact that $\tilde{\mathcal{M}}_{\infty}$ is well-founded follows from the next lemma.

Lemma 3.17 (Lemma 5.10 in [12]). $\tilde{\mathcal{M}}_{\infty}=\tilde{\mathcal{M}}_{\infty}^{+}$.

The second direct limit system. To obtain HOD of some inner model from the direct limit, we in particular need to show that the direct limit is in fact contained in HOD of that inner model. In our setting we therefore need to internalize the direct limit system into the inner model $M_{n}(x)[g]$ fixed above. We first aim to define a direct limit system similar to $\left(\tilde{\mathcal{F}}, \leq_{\tilde{\mathcal{I}}}\right)$ in $M_{n}(x)$ analogous to [12]. In a second step, we then modify the system to obtain direct limit systems with the same direct limit which are definable in $M_{n}(x)[g]$. 
The notion of $n$-suitability from Definition 3.2 is already internal to $M_{n}(x)$ and $M_{n}(x)[g]$, i.e., if $\mathcal{N} \in M_{n}(x) \mid \kappa$ then $\mathcal{N}$ is $n$-suitable in $V$ iff $\mathcal{N}$ is $n$-suitable in $M_{n}(x)$ by the following lemma.

Lemma 3.18. Let $\delta_{0}$ denote the least Woodin cardinal in $M_{n}(x)$.

(1) For all $y \in V_{\delta_{0}}^{M_{n}(x)[g]}, L p^{n}(y) \in \operatorname{HOD}_{y}^{M_{n}(x)[g]}$.

(2) $V_{\delta_{0}}^{M_{n}(x)}$ and $V_{\delta_{0}}^{M_{n}(x)[g]}$ are closed under the operation $y \mapsto L p^{n}(y)$.

Proof. Let $y \in V_{\delta_{0}}^{M_{n}(x)[g]}$ be arbitrary. The model $M_{n}(x)[g]$ can be organized as a $V_{\kappa}^{M_{n}(x)[g]}$-premouse and as such it inherits the iterability from $M_{n}(x)$ and is in fact equal to $M_{n}\left(V_{\kappa}^{M_{n}(x)[g]}\right)$. Consider $L[E](y)^{M_{n}(x)[g]}$, the result of a fully backgrounded extender construction above $y$ using extenders from the sequence of $M_{n}(x)[g]$ organized as a $V_{\kappa}^{M_{n}(x)[g]}$-premouse, and compare it with $L p^{n}(y)$. First, we argue that $L p^{n}(y)$ does not move. If it would move, the $L p^{n}(y)$-side of the coiteration would have to drop because $L p^{n}(y)$ does not have any total extenders. Moreover, it would have to iterate to a proper class model which is equal to an iterate of $L[E](y)^{M_{n}(x)[g]}$. As $L[E](y)^{M_{n}(x)[g]}$ has $n$ Woodin cardinals, this would imply that $L p^{n}(y)$ has a level which is not $n$-small, contradicting the definition of $L p^{n}(y)$.

Therefore, $L p^{n}(y) \triangleleft \mathcal{R}$ for some iterate $\mathcal{R}$ of $L[E](y)^{M_{n}(x)[g]}{ }^{5}$ The iteration from $L[E](y)^{M_{n}(x)[g]}$ to $\mathcal{R}$ resulting from the comparison process can be defined over $L[E](y)^{M_{n}(x)[g]}$ from the extender sequence of $L[E](y)^{M_{n}(x)[g]}$ and a finite sequence of ordinals as it cannot leave any total measures behind and thus can only use measures of order 0 . Let $K\left(V_{\kappa}^{M_{n}(x)[g]}\right)^{M_{n}\left(V_{\kappa}^{M_{n}(x)[g]}\right)}$ denote the core model constructed above $V_{\kappa}^{M_{n}(x)[g]}$ inside $M_{n}\left(V_{\kappa}^{M_{n}(x)[g]}\right)$, an $n$-small premouse with $n$ Woodin cardinals, in the sense of [14]. Then $K\left(V_{\kappa}^{M_{n}(x)[g]}\right)^{M_{n}\left(V_{\kappa}^{M_{n}(x)[g]}\right)}=$ $M_{n}\left(V_{\kappa}^{M_{n}(x)[g]}\right)$ by [14, Lemma 1.1 (due to Steel)]. Recall that the reorganization of $M_{n}(x)[g]$ as a $V_{\kappa}^{M_{n}(x)[g]}$-premouse is equal to $M_{n}\left(V_{\kappa}^{M_{n}(x)[g]}\right)$. Hence, the extender sequence of the $V_{\kappa}^{M_{n}(x)[g]}$-premouse $M_{n}(x)[g]$ is in $\mathrm{OD}_{V_{\kappa}^{M_{n}(x)[g]}}^{M_{n}(x)[g]}=\mathrm{OD}^{M_{n}(x)[g]}$. Therefore $L p^{n}(y) \in \operatorname{HOD}^{M_{n}(x)[g]}$ by the definability of the $L[E]$-construction.

For (2), the closure of $V_{\delta_{0}}^{M_{n}(x)[g]}$ follows immediately from (1). For $V_{\delta_{0}}^{M_{n}(x)}$ notice that for $y \in V_{\delta_{0}}^{M_{n}(x)}, L p^{n}(y) \in \operatorname{HOD}_{y}^{M_{n}(x)[g]} \subseteq \operatorname{HOD}_{y}^{M_{n}(x)}$ by homogeneity of the forcing.

By stacking the $L p^{n}$-operation, the uniform proof of Lemma 3.18 in fact shows that for all $y \in V_{\delta_{0}}^{M_{n}(x)}, M_{n}(y) \mid \kappa_{0} \in \operatorname{HOD}_{y}^{M_{n}(x)[g]}$, where $\kappa_{0}$ denotes the least measurable cardinal in $M_{n}(y)$.

The definitions of short tree, maximal tree, and correctly guided finite stack we gave above are internal to $M_{n}(x)$ and $M_{n}(x)[g]$ as well, as they can be defined only using the $L p^{n}$-operation. The only notion we have to take care of is $s$-iterability since

\footnotetext{
${ }^{5}$ In fact, it is not hard to see that $\mathcal{R}=L[E](y)^{M_{n}(x)[g]}$ and hence $L p^{n}(y) \triangleleft L[E](y)^{M_{n}(x)[g]}$ but we will not need this observation.
} 
it is not even clear how the sets $T_{s, k}^{\mathcal{N}}$ can be identified inside $M_{n}(x)$. This obstacle is solved by shrinking the direct limit $\operatorname{system}\left(\tilde{\mathcal{F}}, \leq_{\tilde{\mathcal{I}}}\right)$ to a dense subset as follows.

DEFINITION 3.19. Let

$$
\begin{aligned}
& \mathcal{G}=\left\{\mathcal{N} \in M_{n}(x)|\kappa| \mathcal{N} \text { is } n \text {-suitable and } M_{n}(x) \vDash\right. \text { "for some cardinal } \\
& \text { cutpoint } \eta, \delta^{\mathcal{N}}=\eta^{+}, \mathcal{N}\left|\delta^{\mathcal{N}} \in \mathcal{P}\left(M_{n}(x) \mid \eta^{+}\right) \cap M_{n}(x)\right|\left(\eta^{+}+\omega\right), \\
& \text { and } M_{n}(x) \mid \eta \text { is generic over } \mathcal{N} \text { for the } \delta^{\mathcal{N}} \text {-generator version of } \\
& \text { the extender algebra at } \left.\delta^{\mathcal{N}} \text { " }\right\}
\end{aligned}
$$

See for example Section 4.1 in [2] for an introduction to the $\delta$-generator version of the extender algebra at some Woodin cardinal $\delta$. The following lemma shows how we can use the fact that $\mathcal{N} \in \mathcal{G}$ to detect $M_{n}\left(\mathcal{N} \mid \delta^{\mathcal{N}}\right)$ inside $M_{n}(x)$. For some premouse $\mathcal{R} \in \mathcal{G}$ we denote the last model of a $\mathcal{P}$-construction above $\mathcal{R} \mid \delta^{\mathcal{R}}$ performed inside $M_{n}(x)$ as introduced in [15] (see also Proposition 2.3 and Definition 2.4 in [12]) by $\mathcal{P}^{M_{n}(x)}\left(\mathcal{R} \mid \delta^{\mathcal{R}}\right)$.

Lemma 3.20 (Lemma 5.11 in [12]). Let $\mathcal{N} \in M_{n}(x) \mid \kappa$ be an $n$-suitable premouse such that for some cardinal cutpoint $\eta<\delta^{\mathcal{N}}$ of $M_{n}(x)$, we have that $\mathcal{N} \mid \delta^{\mathcal{N}} \in$ $\mathcal{P}\left(M_{n}(x) \mid \eta^{+}\right) \cap M_{n}(x) \mid\left(\eta^{+}+\omega\right)$ and $M_{n}(x) \mid \eta$ is generic over $\mathcal{N}$ for the $\delta^{\mathcal{N}}$-generator version of the extender algebra at $\delta^{\mathcal{N}}$. Then $\mathcal{N} \in \mathcal{G}$ and

$$
\mathcal{P}^{M_{n}(x)}\left(\mathcal{N} \mid \delta^{\mathcal{N}}\right)=M_{n}\left(\mathcal{N} \mid \delta^{\mathcal{N}}\right) .
$$

In particular, $M_{n}\left(\mathcal{N} \mid \delta^{\mathcal{N}}\right)\left[M_{n}(x) \mid \eta\right]=M_{n}(x)$.

Using pseudo-genericity iterations (see Lemma 3.9) we can obtain the following corollary.

COROLlary 3.21. Let $\mathcal{N}$ be a short tree iterable n-suitable premouse such that $\mathcal{N} \in M_{n}(x) \mid \kappa$. Then there is a correctly guided finite stack on $\mathcal{N}$ with last model $\mathcal{M}$ such that $\mathcal{M} \in \mathcal{G}$ and $\mathcal{P}^{M_{n}(x)}\left(\mathcal{M} \mid \delta^{\mathcal{M}}\right)=M_{n}\left(\mathcal{M} \mid \delta^{\mathcal{M}}\right)$.

Now the following definition of $s$-iterability agrees with the previous one given in Definition 3.10 for $n$-suitable premice in $\mathcal{G}$.

Definition 3.22. For $\mathcal{N} \in \mathcal{G}, s \in[\text { Ord }]^{<\omega}$, and $k<\omega$ let

$$
\begin{array}{r}
T_{s, k}^{\mathcal{N}, *}=\left\{(t,\ulcorner\phi\urcorner) \in\left[\left(\left(\delta^{\mathcal{N}}\right)^{+k}\right)^{\mathcal{N}}\right]^{<\omega} \times \omega \mid \phi \text { is a } \Sigma_{1}\right. \text {-formula and } \\
\left.\mathcal{P}^{M_{n}(x)}\left(\mathcal{N} \mid \delta^{\mathcal{N}}\right) \vDash \phi[t, s]\right\} .
\end{array}
$$

Then we say for $\mathcal{N} \in \mathcal{G}$ and $s \in[\text { Ord }]^{<\omega}$ that $M_{n}(x) \vDash$ " $\mathcal{N}$ is $s$-iterable below $\kappa$ " iff for every $\operatorname{Col}(\omega,<\kappa)$-generic $G$ over $M_{n}(x)$ and every correctly guided finite stack $\overrightarrow{\mathcal{T}}=\left(\mathcal{T}_{i}, \mathcal{N}_{i} \mid i \leq m\right) \in \mathrm{HC}^{M_{n}(x)[G]}$ on $\mathcal{N}$ with last model $\mathcal{M} \in \mathcal{G}$, there is a sequence of branches $\overline{\vec{b}}=\left(b_{i} \mid i \leq m\right) \in M_{n}(x)[G]$ and a sequence of embeddings $\left(\pi_{i} \mid i \leq m\right)$ satisfying $(i)-(i i i)$ in Definition 3.10 such that if we let $\pi_{\overrightarrow{\mathcal{T}}, \vec{b}}=\pi_{m}$ 。 $\pi_{m-1} \circ \cdots \circ \pi_{0}$, then for every $k<\omega$,

$$
\pi_{\overrightarrow{\mathcal{T}}, \vec{b}}\left(T_{s, k}^{\mathcal{N}, *}\right)=T_{s, k}^{\mathcal{M}, *} .
$$

In addition, we define $M_{n}(x) \vDash$ " $\mathcal{N}$ is strongly $s$-iterable below $\kappa$ " analogous to Definition 3.12 for all $\operatorname{Col}(\omega,<\kappa)$-generic $G$ and stacks $\overrightarrow{\mathcal{T}}, \overrightarrow{\mathcal{U}} \in M_{n}(x)[G]$. For $\mathcal{N} \in \mathcal{G}$, 
$s \in[\text { Ord }]^{<\omega}$, and $k<\omega$, we have $T_{s, k}^{\mathcal{N}, *}=T_{s, k}^{\mathcal{N}}$, so we will omit the $*$ for $\mathcal{N} \in \mathcal{G}$. Using this, $\gamma_{s}^{\mathcal{N}}$ and $H_{s}^{\mathcal{N}}$ are defined as before. Then we can define the internal direct limit system as follows.

DeFinition 3.23. Let

$$
\begin{aligned}
& \mathcal{I}=\left\{(\mathcal{N}, s) \mid \mathcal{N} \in \mathcal{G}, s \in[\text { Ord }]^{<\omega},\right. \text { and } \\
& M_{n}(x)\vDash " \mathcal{N} \text { is strongly } s \text {-iterable below } \kappa "\}
\end{aligned}
$$

and

$$
\mathcal{F}=\left\{H_{s}^{\mathcal{N}} \mid(\mathcal{N}, s) \in \mathcal{I}\right\} .
$$

Moreover, for $(\mathcal{N}, s),(\mathcal{M}, t) \in \mathcal{I}$ we let $(\mathcal{N}, s) \leq(\mathcal{M}, t)$ iff there is a correctly guided finite stack on $\mathcal{N}$ with last model $\mathcal{M}$ and $s \subseteq t$. In this case we let as before $\pi_{(\mathcal{N}, s),(\mathcal{M}, t)}: H_{s}^{\mathcal{N}} \rightarrow H_{t}^{\mathcal{M}}$ denote the canonical corresponding embedding.

For clarity, we sometimes write $\leq_{\mathcal{I}}$ for $\leq$. Similar as before we have that for every $\mathcal{N} \in \mathcal{G}$ and $s \in$ [Ord] ${ }^{<\omega}$ there is a normal correct iterate $\mathcal{M}$ of $\mathcal{N}$ such that $(\mathcal{M}, s) \in \mathcal{I}$. Using the fact that $\kappa$ is inaccessible and a limit of cutpoints in $M_{n}(x)$ we can obtain the following lemma.

Lemma 3.24 (Lemma 5.14 in [12]). $\leq$ is directed.

Therefore we can again define the direct limit.

Definition 3.25. Let $\mathcal{M}_{\infty}$ be the direct limit of $(\mathcal{F}, \leq)$ under the embeddings $\pi_{(\mathcal{N}, s),(\mathcal{M}, t)}$. Moreover, let $\delta_{\infty}=\delta^{\mathcal{M}_{\infty}}$ be the Woodin cardinal in $\mathcal{M}_{\infty}$ and $\pi_{(\mathcal{N}, s), \infty}$ : $H_{s}^{\mathcal{N}} \rightarrow \mathcal{M}_{\infty}$ be the direct limit embedding for all $(\mathcal{N}, s) \in \mathcal{I}$.

An argument similar to the one for Lemma 3.17 shows that this direct limit is well-founded as well. As we will use ideas from this proof in the next section, we will give some details here. We again first define another direct limit system which consists of iterates of $M_{n+1}^{-}$and then show that its direct limit $\mathcal{M}_{\infty}^{+}$is equal to $\mathcal{M}_{\infty}$.

Definition 3.26. Let

$$
\begin{gathered}
\mathcal{F}^{+}=\{\mathcal{Q} \in \mathcal{G} \mid \mathcal{Q} \text { is the last model of a correctly guided } \\
\text { finite stack on } \left.M_{n+1}^{-} \text {via } \Sigma_{M_{n+1}^{-}}\right\} .
\end{gathered}
$$

Moreover, let $\mathcal{P} \leq{ }^{+} \mathcal{Q}$ for $\mathcal{P}, \mathcal{Q} \in \mathcal{F}^{+}$iff there is a correctly guided finite stack on $\mathcal{P}$ according to the tail strategy $\Sigma_{\mathcal{P}}$ with last model $\mathcal{Q}$. In this case we let $i_{\mathcal{P}, \mathcal{Q}}: \mathcal{P} \rightarrow \mathcal{Q}$ denote the corresponding iteration embedding.

Then $\leq^{+}$on $\mathcal{F}^{+}$is directed, so we can define the direct limit.

Definition 3.27. Let $\mathcal{M}_{\infty}^{+}$be the direct limit of $\left(\mathcal{F}^{+}, \leq^{+}\right)$under the embeddings $i_{\mathcal{P}, \mathcal{Q}}$. Moreover, let $i_{\mathcal{Q}, \infty}: \mathcal{Q} \rightarrow \mathcal{M}_{\infty}^{+}$denote the direct limit embedding for all $\mathcal{Q} \in \mathcal{F}^{+}$.

Then it is easy to see that $\mathcal{M}_{\infty}^{+}$is well-founded as $\mathcal{F}^{+}$only consists of iterates of $M_{n+1}^{-}$according to the canonical iteration strategy $\Sigma_{M_{n+1}^{-}}$.

Lemma 3.28 (Lemma 5.15 in [12]). $\mathcal{M}_{\infty}^{+}=\mathcal{M}_{\infty}$ and hence $\mathcal{M}_{\infty}$ is well-founded . 
Proof. We construct a sequence $\left(\mathcal{Q}_{i} \mid i<\omega\right)$ of iterates of $M_{n+1}^{-}$such that $\mathcal{Q}_{i} \in$ $\mathcal{F}^{+}$for every $i<\omega$ and $\left(\mathcal{Q}_{i} \mid i<\omega\right)$ is cofinal in $\mathcal{G}$, i.e., for every $\mathcal{N} \in \mathcal{G}$ there is an $i<\omega$ such that $\mathcal{Q}_{i}$ is the last model of a correctly guided finite stack on $\mathcal{N}$.

In $V$, fix some sequence $\left(\xi_{i} \mid i<\omega\right)$ of ordinals cofinal in $\kappa$. We define $\left(\mathcal{Q}_{i} \mid i<\omega\right)$ together with a strictly increasing sequence $\left(\eta_{i} \mid i<\omega\right)$ of cardinal cutpoints of $M_{n}(x) \mid \kappa$ by induction on $i<\omega$. So let $\mathcal{Q}_{0}=M_{n+1}^{-}$and let $\eta_{0}<\kappa$ be a cardinal cutpoint of $M_{n}(x)$. Moreover assume that we already constructed $\left(\mathcal{Q}_{i} \mid i \leq j\right)$ and $\left(\eta_{i} \mid i \leq j\right)$ with the above mentioned properties such that in addition $\left(\mathcal{Q}_{i} \mid i \leq\right.$ $j) \in M_{n}(x) \mid \eta_{j}$. Let $\mathcal{Q}_{j+1}^{*}$ be the result of simultaneously pseudo-comparing (in the sense of Lemma 3.8) all $n$-suitable premice $\mathcal{M}$ such that $\mathcal{M} \in \mathcal{G} \cap M_{n}(x) \mid \eta_{j}$. Then in particular $\mathcal{Q}_{j+1}^{*}$ is a normal iterate of $\mathcal{Q}_{j}$ according to the canonical tail iteration strategy $\Sigma_{\mathcal{Q}_{j}}$, but $\mathcal{Q}_{j+1}^{*}$ might not be in $\mathcal{G}$. Let $v$ be a cardinal cutpoint of $M_{n}(x)$ such that $\eta_{j}<v<\kappa$ and $\mathcal{Q}_{j+1}^{*} \in M_{n}(x) \mid v$. Note that such a $v$ exists as $\kappa$ is inaccessible and a limit of cardinal cutpoints in $M_{n}(x)$. Let $\mathcal{Q}_{j+1}$ be the normal iterate of $\mathcal{Q}_{j+1}^{*}$ according to the canonical tail strategy $\Sigma_{\mathcal{Q}_{j+1}^{*}}$ of $\Sigma_{\mathcal{Q}_{j}}$ obtained by Woodin's genericity iteration such that $M_{n}(x) \mid v$ is generic over $\mathcal{Q}_{j+1}$ for the $\delta^{\mathcal{Q}_{j+1}}$ generator version of the extender algebra (see for example Section 4.1 in [2]). Then $\mathcal{Q}_{j+1} \in \mathcal{G}$ is as desired. Finally choose $\eta_{j+1}<\kappa$ such that $\eta_{j+1}>\max \left(\eta_{j}, \xi_{j}\right), \eta_{j+1}$ is a cardinal cutpoint in $M_{n}(x)$ and $\left(\mathcal{Q}_{i} \mid i \leq j+1\right) \in M_{n}(x) \mid \eta_{j+1}$.

Now we define an embedding $\sigma: \mathcal{M}_{\infty} \rightarrow \mathcal{M}_{\infty}^{+}$as follows. Let $x \in \mathcal{M}_{\infty}$. Since $\left(\mathcal{Q}_{i} \mid i<\omega\right)$ is cofinal in $\mathcal{G}$, there are $i, m<\omega$ such that $\left(\mathcal{Q}_{i}, s_{m}\right) \in \mathcal{I}$ and $x=$ $\pi_{\left(\mathcal{Q}_{i}, s_{m}\right), \infty}(\bar{x})$ for some $\bar{x} \in H_{s_{m}}^{\mathcal{Q}_{i}} \subseteq \mathcal{Q}_{i}$. Then we let $\sigma(x)=i_{\mathcal{Q}_{i}, \infty}(\bar{x})$.

It follows as in the proof of Lemma 5.10 in [12] that the definition of $\sigma$ does not depend on the choice of $i, m<\omega$ and in fact $\sigma=\mathrm{id}$.

Moreover, it is possible to compute $\delta_{\infty}$.

LEMMA 3.29 (Lemma 5.16 in [12]). $\delta_{\infty}=\left(\kappa^{+}\right)^{M_{n}(x)}$.

Direct limit systems in $\operatorname{HOD}^{M_{n}(x)[g]}$. Finally, we will argue that $\mathcal{M}_{\infty} \in$ $\operatorname{HOD}^{M_{n}(x)[g]}$ by first defining direct limit systems in various premice $M(y)$ satisfying certain properties definable in $M_{n}(x)[g]$ and then showing that the direct limits $\mathcal{M}_{\infty}^{M(y)}$ are equal to $\mathcal{M}_{\infty}$. A similar approach but in a completely different setting can be found in [13].

In what follows, we will let $(K(z))^{N}$ denote the core model constructed above a real $z$ inside some $n$-small model $N$ with $n$ Woodin cardinals in the sense of [14], i.e., the core model $K(z)$ is constructed between consecutive Woodin cardinals. Lemma 1.1 in [14] (due to Steel) implies that $(K(x))^{M_{n}(x)}=M_{n}(x)$. We will use this fact and consider more arbitrary premice with this property in what follows. We state the following definitions in $V$, but we will later apply them inside $M_{n}(x)[g]$.

Definition 3.30. Let $y \in{ }^{\omega} \omega \cap M_{n}(x)[g]$. Then we say $y$ is pre-dlm-suitable iff there is a proper class $y$-premouse $M(y)$ satisfying the following properties.

(i) $M(y)$ is $n$-small and has $n$ Woodin cardinals,

(ii) the least inaccessible cardinal in $M(y)$ is $\kappa$,

(iii) $M(y)=(K(y))^{M(y)}$, and 
(iv) there is a $\operatorname{Col}(\omega,<\kappa)$-generic $h$ over $M(y)$ such that

$$
M(y)[h]=M_{n}(x)[g] .
$$

We also call such a $y$-premouse $M(y)$ pre-dlm-suitable and say that $M(y)$ witnesses that $y$ is pre-dlm-suitable.

Using this, we can define a version of the direct limit system $\mathcal{F}$ inside arbitrary pre-dlm-suitable $y$-premice $M(y)$.

Definition 3.31. Let $y \in{ }^{\omega} \omega$ be pre-dlm-suitable as witnessed by $M(y)$. Then we let

$$
\begin{gathered}
\mathcal{G}^{M(y)}=\{\mathcal{N} \in M(y)|\kappa| \mathcal{N} \text { is } n \text {-suitable and } M(y) \vDash \text { "for some cardinal } \\
\text { cutpoint } \eta, \delta^{\mathcal{N}}=\eta^{+}, \mathcal{N}\left|\delta^{\mathcal{N}} \in \mathcal{P}\left(M(y) \mid \eta^{+}\right) \cap M(y)\right|\left(\eta^{+}+\omega\right), \\
\text { and } M(y) \mid \eta \text { is generic over } \mathcal{N} \text { for the } \delta^{\mathcal{N}} \text {-generator version of } \\
\text { the extender algebra at } \left.\delta^{\mathcal{N} "}\right\} .
\end{gathered}
$$

Analogous as before, we can now define when for an $n$-suitable premouse $\mathcal{N}$, $M(y) \vDash " \mathcal{N}$ is strongly $s$-iterable below $\kappa$ " by referring to $\mathcal{P}^{M(y)}\left(\mathcal{N} \mid \delta^{\mathcal{N}}\right)$ in the definition of $\left(T_{s, k}^{\mathcal{N}, *}\right)^{M(y)}$. Let $\gamma_{s}^{\mathcal{N}, M(y)}$ and $H_{s}^{\mathcal{N}, M(y)}$ be defined analogous to $\gamma_{s}^{\mathcal{N}}$ and $H_{s}^{\mathcal{N}}$ inside $M(y)$ using $\left(T_{s, k}^{\mathcal{N}, *}\right)^{M(y)}$. For $M(y)=M_{n}(x)$ and $\mathcal{N} \in \mathcal{G}$ this agrees with our previous definition of strong $s$-iterability.

Definition 3.32. Let $y \in{ }^{\omega} \omega$ be pre-dlm-suitable as witnessed by $M(y)$. Then we let

$$
\begin{gathered}
\mathcal{I}^{M(y)}=\left\{(\mathcal{N}, s) \mid \mathcal{N} \in \mathcal{G}^{M(y)}, s \in[\text { Ord }]^{<\omega},\right. \text { and } \\
M(y) \vDash “ \mathcal{N} \text { is strongly } s \text {-iterable below } \kappa "\}
\end{gathered}
$$

and

$$
\mathcal{F}^{M(y)}=\left\{H_{s}^{\mathcal{N}, M(y)} \mid(\mathcal{N}, s) \in \mathcal{I}^{M(y)}\right\} .
$$

Moreover, for $(\mathcal{N}, s),(\mathcal{M}, t) \in \mathcal{I}^{M(y)}$ we let $(\mathcal{N}, s) \leq_{\mathcal{I}^{M(y)}}(\mathcal{M}, t)$ iff there is a correctly guided finite stack on $\mathcal{N}$ with last model $\mathcal{M}$ and $s \subseteq t$. In this case we let $\pi_{(\mathcal{N}, s),(\mathcal{M}, t)}^{M(y)}: H_{s}^{\mathcal{N}, M(y)} \rightarrow H_{t}^{\mathcal{M}, M(y)}$ denote the canonical corresponding embedding. Finally, let $\mathcal{M}_{\infty}^{M(y)}$ denote the direct limit of $\left(\mathcal{F}^{M(y)}, \leq_{\mathcal{I}^{M(y)}}\right)$ under these embeddings.

We will now strengthen this and define when a real $y \in{ }^{\omega} \omega$ (or a $y$-premouse $M(y))$ is dlm-suitable.

Definition 3.33. Let $y \in{ }^{\omega} \omega \cap M_{n}(x)[g]$ be pre-dlm-suitable as witnessed by some $y$-premouse $M(y)$. We say that $y$ is dlm-suitable (witnessed by $M(y)$ ) iff

(i) for every $s \in[\text { Ord }]^{<\omega}$ there is a premouse $\mathcal{N}$ such that $(\mathcal{N}, s) \in \mathcal{I}^{M(y)}$ and

(ii) for every $\mathcal{N} \in \mathcal{G}^{M(y)}$,

$$
\mathcal{P}^{M(y)}\left(\mathcal{N} \mid \delta^{\mathcal{N}}\right)=K^{M_{n}(x)[g]}\left(\mathcal{N} \mid \delta^{\mathcal{N}}\right) .
$$

LeMma 3.34. $M_{n}(x)$ witnesses that $x$ is dlm-suitable. 
Proof. The fact that $M_{n}(x)$ satisfies (i) follows from Lemma 3.13 and Corollary 3.21, so we only have to show (ii). Let $\mathcal{N} \in \mathcal{G}$. Then $\mathcal{P}^{M_{n}(x)}\left(\mathcal{N} \mid \delta^{\mathcal{N}}\right)=M_{n}\left(\mathcal{N} \mid \delta^{\mathcal{N}}\right)$ by Lemma 3.20. Moreover, there is some $G$ generic over the result of the $\mathcal{P}$-construction $\mathcal{P}^{M_{n}(x)}\left(\mathcal{N} \mid \delta^{\mathcal{N}}\right)$ for the $\delta^{\mathcal{N}}$-generator version of the extender algebra at $\delta^{\mathcal{N}}$ with $\mathcal{P}^{M_{n}(x)}\left(\mathcal{N} \mid \delta^{\mathcal{N}}\right)[G]=M_{n}(x)$. That means

$$
M_{n}\left(\mathcal{N} \mid \delta^{\mathcal{N}}\right)[G]=M_{n}(x) .
$$

Now,

$$
\begin{aligned}
K^{M_{n}(x)[g]}\left(\mathcal{N} \mid \delta^{\mathcal{N}}\right) & =K^{M_{n}\left(\mathcal{N} \mid \delta^{\mathcal{N}}\right)[G][g]}\left(\mathcal{N} \mid \delta^{\mathcal{N}}\right)=K^{M_{n}\left(\mathcal{N} \mid \delta^{\mathcal{N}}\right)}\left(\mathcal{N} \mid \delta^{\mathcal{N}}\right) \\
& =M_{n}\left(\mathcal{N} \mid \delta^{\mathcal{N}}\right)=\mathcal{P}^{M_{n}(x)}\left(\mathcal{N} \mid \delta^{\mathcal{N}}\right)
\end{aligned}
$$

by generic absoluteness of the core model and Lemma 1.1 in [14] (due to Steel). $\dashv$

Condition $(i i)$ in Definition 3.33 will ensure that for any dlm-suitable $y$-premouse $M(y)$ and $(\mathcal{N}, s),(\mathcal{M}, t) \in \mathcal{I} \cap \mathcal{I}^{M(y)}$ with $(\mathcal{N}, s) \leq_{\mathcal{I}}(\mathcal{M}, t)$ and $(\mathcal{N}, s) \leq_{\mathcal{I}^{M(y)}}$ $(\mathcal{M}, t)$, the induced embeddings $\pi_{(\mathcal{N}, s),(\mathcal{M}, t)}$ and $\pi_{(\mathcal{N}, s),(\mathcal{M}, t)}^{M(y)}$ agree. Hence we can show in the following lemma that the direct limit $\mathcal{M}_{\infty}^{M(y)}$ defined inside some dlmsuitable $M(y)$ will in fact be the same as the direct limit $\mathcal{M}_{\infty}$ defined inside $M_{n}(x)$.

LemMA 3.35. Let $y \in{ }^{\omega} \omega$ be dlm-suitable as witnessed by $M(y)$. Then $\mathcal{F}$ and $\mathcal{F}^{M(y)}$ have cofinally many points in common and $\mathcal{M}_{\infty}=\mathcal{M}_{\infty}^{M(y)}$.

Proof. Let $h$ be $\operatorname{Col}(\omega,<\kappa)$-generic over $M(y)$ such that $M(y)[h]=M_{n}(x)[g]$. Let $(\mathcal{N}, s) \in \mathcal{I}$ and $\left(\mathcal{N}^{\prime}, s^{\prime}\right) \in \mathcal{I}^{M(y)}$. We aim to show that there is some $(\mathcal{M}, t) \in$ $\mathcal{I} \cap \mathcal{I}^{M(y)}$ such that $(\mathcal{N}, s) \leq_{\mathcal{I}}(\mathcal{M}, t)$ and $\left(\mathcal{N}^{\prime}, s^{\prime}\right) \leq_{\mathcal{I}^{M(y)}}(\mathcal{M}, t)$. As condition (ii) in Definition 3.33 yields that the embeddings associated to $\mathcal{F}$ and $\mathcal{F}^{M(y)}$ agree, this suffices to show that $\mathcal{M}_{\infty}=\mathcal{M}_{\infty}^{M(y)}$.

Let $t=s \cup s^{\prime}$. By assumption, there is a $t$-iterable premouse $\mathcal{R}$ in $M_{n}(x)$ and a $t$-iterable premouse $\mathcal{R}^{\prime}$ in $M(y)$. Therefore we can assume that $\mathcal{N}$ and $\mathcal{N}^{\prime}$ are both $t$-iterable in $M_{n}(x)$ and $M(y)$ respectively as we can replace them by the result of their coiteration with $\mathcal{R}$ and $\mathcal{R}^{\prime}$ respectively.

By the choice of $M(y)$ and generic absoluteness of the core model we have

$$
\begin{aligned}
M(y) & =(K(y))^{M(y)}=(K(y))^{M(y)[h]} \\
& =(K(y))^{M_{n}(x)[g]}=(K(y))^{M_{n}(x)[g\lceil\xi]},
\end{aligned}
$$

where $\xi<\kappa$ is such that $y \in M_{n}(x)[g\lceil\xi]$. Analogously, using Lemma 1.1 in [14] due to Steel and generic absoluteness of the core model again,

$$
\begin{aligned}
M_{n}(x) & =(K(x))^{M_{n}(x)}=(K(x))^{M_{n}(x)[g]} \\
& =(K(x))^{M(y)[h]}=(K(x))^{M(y)\left[h\left\lceil\xi^{\prime}\right]\right.},
\end{aligned}
$$

where $\xi^{\prime}<\kappa$ is such that $x \in M(y)\left[h\left\lceil\xi^{\prime}\right]\right.$. Now we can obtain the following claim.

Claim 1. $M(y)$ and $M_{n}(x)$ have cofinally many common cardinal cutpoints below $\kappa$.

Proof. As $M(y)=K(y)^{M_{n}(x)[g \mid \xi]}$ is an inner model of $M_{n}(x)[g\lceil\xi]$, every cardinal above $\xi$ in $M_{n}(x)$ is a cardinal in $M(y)$, so it is easy to find cofinally 
many common cardinals of $M(y)$ and $M_{n}(x)$ below $\kappa$. Recall that $\kappa$ is the least inaccessible cardinal of both $M(y)$ and $M_{n}(x)$. Therefore, every common cardinal of $M(y)$ and $M_{n}(x)$ below $\kappa$ is also a cutpoint of both models.

Moreover, Equations (1) and (2) yield

$$
M(y) \subseteq M_{n}(x)[g\lceil\xi] \subseteq M(y)[h\lceil\zeta],
$$

where $\xi^{\prime}<\zeta<\kappa$ is such that $g\lceil\xi \in M(y)[h\lceil\zeta]$. By the intermediate model theorem (see for example Lemma 15.43 in [4]) this implies that $M_{n}(x)[g\lceil\xi]$ is a generic extension of $M(y)$ for a forcing of size less than $\kappa{ }^{6}$ Since $M_{n}(x)[g\lceil\xi]$ is a generic extension of $M_{n}(x)$ for a forcing of size less than $\kappa$ as well, this implies by Theorem 1.3 in [24] that there is some common inner model $W \subseteq M_{n}(x) \cap M(y)$ such that $M_{n}(x)[g\lceil\xi]$ is a generic extension of $W$ for a forcing of size less than $\kappa$.

As every generic extension via a forcing of size less than $\kappa$ can be absorbed by the collapse of some ordinal $\beta<\kappa$, this yields that we can fix some ordinal $\beta<\kappa$ and some $\operatorname{Col}(\omega, \beta)$-generic $b \in M_{n}(x)[g]$ over $W$ such that $x, y, \mathcal{N}, \mathcal{N}^{\prime} \in W[b]$. Then $M_{n}(x)$ and $M(y)$ exist in $W[b]$ as definable subclasses because

$$
(K(x))^{W[b]}=(K(x))^{M_{n}(x)[g\lceil\xi]}=(K(x))^{M_{n}(x)}=M_{n}(x)
$$

and similarly

$$
(K(y))^{W[b]}=(K(y))^{M_{n}(x)[g \mid \xi \xi]}=(K(y))^{M_{n}(x)[g]}=(K(y))^{M(y)[h]}=M(y)
$$

by generic absoluteness of the core model again. Let $\dot{x}, \dot{y}, \dot{\mathcal{N}}$ and $\dot{\mathcal{N}}^{\prime}$ be $\operatorname{Col}(\omega, \beta)$ names for $x, y, \mathcal{N}$ and $\mathcal{N}^{\prime}$ in $W$. Moreover, let $p \in \operatorname{Col}(\omega, \beta)$ force all properties we need about $\dot{x}, \dot{y}, \dot{\mathcal{N}}$ and $\dot{\mathcal{N}}^{\prime}$. For $q \leq_{\operatorname{Col}(\omega, \beta)} p$ let $b_{q}$ be the $\operatorname{Col}(\omega, \beta)$-generic filter over $W$ such that $\bigcup b_{q}$ agrees with $q$ on $\operatorname{dom}(q)$ and with $\bigcup b$ everywhere else.

Now we construct $(\mathcal{M}, t) \in \mathcal{I} \cap \mathcal{I}^{M(y)}$. Let $\eta<\kappa$ be a cardinal cutpoint of both $M(y)$ and $M_{n}(x)$ such that $\xi, \xi^{\prime}<\eta$, which exists by Claim 1. Then in fact $\left(\eta^{+}\right)^{M_{n}(x)}=\left(\eta^{+}\right)^{M(y)}$ as by Equations (1) and (2) at the beginning of the proof

$$
\left(\eta^{+}\right)^{M(y)} \leq\left(\eta^{+}\right)^{M_{n}(x)[g \mid \xi]}=\left(\eta^{+}\right)^{M_{n}(x)} \leq\left(\eta^{+}\right)^{M(y)\left[h \mid \xi^{\prime}\right]}=\left(\eta^{+}\right)^{M(y)} .
$$

By the same argument, $\left(\eta^{+}\right)^{K\left(\dot{x}^{b q}\right)}=\left(\eta^{+}\right)^{K\left(\dot{y}^{b q}\right)}$ for all $q \leq_{\operatorname{Col}(\omega, \beta)} p$.

Work in $W[b]$. Using Lemmas 3.8 and 3.9, we obtain an inner model $\mathcal{M}$ by pseudo-comparing all $(\dot{\mathcal{N}})^{b_{q}}$ and $\left(\dot{\mathcal{N}}^{\prime}\right)^{b_{q}}$ for $q \leq_{\mathrm{Col}(\omega, \beta)} p$ and simultaneously pseudo-genericity iterating such that $K\left(\dot{x}^{b_{q}}\right) \mid \eta$ and $K\left(\dot{y}^{b_{q}}\right) \mid \eta$ are generic over $\mathcal{M}$ and $\delta^{\mathcal{M}}=\left(\eta^{+}\right)^{K\left(\dot{x}^{b} q\right)}=\left(\eta^{+}\right)^{K\left(\dot{y}^{b} q\right)}$. Since $\mathcal{M}$ is definable in $W[b]$ from $\left\{b_{q} \mid q \leq_{\operatorname{Col}(\omega, \beta)}\right.$ $p\}$ and parameters from $W$, we have that in fact $\mathcal{M} \in W \subseteq M_{n}(x) \cap M(y)$, as $\mathcal{M}$ does not depend on the choice of the generic $b$. Moreover, $\mathcal{M}$ is a correct iterate of $\mathcal{N}$ in $M_{n}(x)$ and a correct iterate of $\mathcal{N}^{\prime}$ in $M(y)$.

As argued above, we can assume that $\mathcal{N}$ and $\mathcal{N}^{\prime}$ are $t$-iterable in $M_{n}(x)$ and $M(y)$ respectively for $t=s \cup s^{\prime}$. Therefore $\mathcal{M}$ is $t$-iterable in both, $M_{n}(x)$ and $M(y)$. Hence, $(\mathcal{M}, t) \in \mathcal{I} \cap \mathcal{I}^{M(y)},(\mathcal{N}, s) \leq_{\mathcal{I}}(\mathcal{M}, t)$, and $\left(\mathcal{N}^{\prime}, s^{\prime}\right) \leq_{\mathcal{I}^{M(y)}}(\mathcal{M}, t)$, as desired.

\footnotetext{
${ }^{6}$ I.e., $M(y)$ is a ground of $M_{n}(x)[g \mid \xi]$. See for example [3] or [24] for an introduction to the theory of grounds.
} 
This yields that $\mathcal{M}_{\infty} \in \operatorname{HOD}^{M_{n}(x)[g]}$.

§4. HOD below $\delta_{\infty}$. In this section we will show that $\mathrm{HOD}^{M_{n}(x)[g]}$ and $\mathcal{M}_{\infty}$ agree up to $\delta_{\infty}$ by generalizing the arguments in Section 3.4 in [23]. We show a version of Woodin's derived model resemblance for our setting. For this, we do not need to talk about generic extensions such as $M(y)[h]$ and work with $M(y)$ directly instead.

We start with expanding our direct limit $\mathcal{M}_{\infty}$ to the proper class premouse $\hat{\mathcal{M}}_{\infty}=$ $M_{n}\left(\mathcal{M}_{\infty} \mid \delta_{\infty}\right)$ and define a direct limit system $\hat{\mathcal{F}}$ of expansions of the elements of $\mathcal{F}$ that converges to $\hat{\mathcal{M}}_{\infty}$. For an $n$-suitable premouse $\mathcal{N}$ and $s \in[\text { Ord }]^{<\omega}$ with $\max (s)$ a uniform indiscernible above the Woodin cardinals in $M_{n}\left(\mathcal{N} \mid \delta^{\mathcal{N}}\right)$, we let $\hat{\mathcal{N}}=M_{n}\left(\mathcal{N} \mid \delta^{\mathcal{N}}\right)$ be the proper class expansion of $\mathcal{N}, s^{-}=s \backslash \max (s)$,

$$
\gamma_{s}^{\hat{\mathcal{N}}}=\sup \left(\operatorname{Hull}_{1}^{\hat{\mathcal{N}} \mid \max (s)}\left(s^{-}\right) \cap \delta^{\mathcal{N}}\right),
$$

and

$$
H_{s}^{\hat{\mathcal{N}}}=\operatorname{Hull}_{1}^{\hat{\mathcal{N}} \mid \max (s)}\left(\gamma_{s}^{\hat{\mathcal{N}}} \cup s^{-}\right) .
$$

Now we let

$$
\hat{\mathcal{F}}=\left\{H_{s}^{\hat{\mathcal{N}}} \mid(\mathcal{N}, s) \in \mathcal{I}\right\}
$$

and for $(\mathcal{N}, s),(\mathcal{M}, t) \in \mathcal{I}$ with $(\mathcal{N}, s) \leq(\mathcal{M}, t)$ we let $\hat{\pi}_{(\mathcal{N}, s),(\mathcal{M}, t)}: H_{s}^{\hat{\mathcal{N}}} \rightarrow H_{t}^{\hat{\mathcal{M}}}$ denote the canonical corresponding embedding extending $\pi_{\left(\mathcal{N}, s^{-}\right),\left(\mathcal{M}, t^{-}\right)}$.

Finally, let $\hat{\mathcal{M}}_{\infty}$ be the direct limit of $(\hat{\mathcal{F}}, \leq)$ under the embeddings $\hat{\pi}_{(\mathcal{N}, s),(\mathcal{M}, t)}$ and let $\hat{\pi}_{(\mathcal{N}, s), \infty}: H_{s}^{\hat{\mathcal{N}}} \rightarrow \hat{\mathcal{M}}_{\infty}$ for $(\mathcal{N}, s) \in \mathcal{I}$ denote the direct limit embedding. Then it is easy to see that $M_{n}\left(\mathcal{M}_{\infty} \mid \delta_{\infty}\right)=\hat{\mathcal{M}}_{\infty}$ as we can define a similar direct limit system $\hat{\mathcal{F}}^{+}$of premice expanding the $n$-suitable premice in $\mathcal{F}^{+}$.

Choose for any ordinal $\alpha$ an arbitrary $(\mathcal{N}, s) \in \mathcal{I}$ such that $\alpha \in s \backslash \max (s)$ and let $\alpha^{*}=\hat{\pi}_{(\mathcal{N}, s), \infty}(\alpha)$. Note that the value of $\alpha^{*}$ does not depend on the choice of $(\mathcal{N}, s)$. We also let $t^{*}=\left\{\alpha^{*} \mid \alpha \in t\right\}$ for $t \in[\text { Ord }]^{<\omega}$.

LEMma 4.1. Let $\mathcal{N}$ be an $n$-suitable premouse and $s \in[\mathrm{Ord}]^{<\omega}$ such that $(\mathcal{N}, s) \in$ I. Let $\bar{\xi}<\gamma_{s}^{\mathcal{N}}, \xi=\pi_{(\mathcal{N}, s), \infty}(\bar{\xi})$ and $t \in[\text { Ord }]^{<\omega}$. Moreover, let $\varphi\left(v_{0}, v_{1}\right)$ be a formula in the language of premice, i.e., we allow the extender sequence as a predicate. Then the following are equivalent.

(a) $M_{n}\left(\mathcal{M}_{\infty} \mid \delta_{\infty}\right) \vDash \varphi\left(\xi, t^{*}\right)$ and

(b) in $M_{n}(x)[g]$, there is some dlm-suitable $y \in{ }^{\omega} \omega$ witnessed by $M(y)$ with $(\mathcal{N}, s) \in \mathcal{I}^{M(y)}$ and a correctly guided finite stack on $\mathcal{N}$ with last model $\mathcal{M} \in M(y)$ such that whenever $\mathcal{R} \in \mathcal{G}^{M(y)}$ is the last model of a correctly guided finite stack on $\mathcal{M}$, then $\mathcal{P}^{M(y)}\left(\mathcal{R} \mid \delta^{\mathcal{R}}\right) \vDash \varphi\left(\pi_{(\mathcal{N}, s),(\mathcal{R}, s)}^{M(y)}(\bar{\xi}), t\right)$.

Proof. To prove that $(a)$ implies $(b)$ we assume toward a contradiction that $(b)$ is false. So in $M_{n}(x)[g]$ for all dlm-suitable $y \in{ }^{\omega} \omega$ and $M(y)$ witnessing this with $(\mathcal{N}, s) \in \mathcal{I}^{M(y)}$ and all correctly guided finite stacks on $\mathcal{N}$ with last model $\mathcal{M} \in M(y)$, there is a correctly guided finite stack on $\mathcal{M}$ with last model $\mathcal{R} \in \mathcal{G}^{M(y)}$ such that $\mathcal{P}^{M(y)}\left(\mathcal{R} \mid \delta^{\mathcal{R}}\right) \vDash \neg \varphi\left(\pi_{(\mathcal{N}, s),(\mathcal{R}, s)}^{M(y)}(\bar{\xi}), t\right)$. 
We can assume without loss of generality that $\mathcal{N} \in M_{n}(x)$ is the last model of a correctly guided finite stack on $M_{n+1}^{-}$via the canonical iteration strategy $\Sigma_{M_{n+1}^{-}}$ and strongly $s$-iterable below $\kappa$. Moreover, we can assume that $\max (s)$ is a uniform indiscernible. If this is not already the case, we replace $\mathcal{N}$ by a pseudo-iterate of the result of the pseudo-comparison of $\mathcal{N}$ with $M_{n+1}^{-}$using Lemma 3.8 and Corollary 3.21 .

CLAIM 1. There are n-suitable premice $\mathcal{N}_{k} \in \mathcal{F}^{+}$for $k<\omega$ which are cofinal in $\mathcal{F}^{+}$ such that $\mathcal{N}_{0}=\mathcal{N}$ and for all $k<\omega$,

$$
M_{n}\left(\mathcal{N}_{k} \mid \delta^{\mathcal{N}_{k}}\right) \vDash \neg \varphi\left(\bar{\xi}_{k}, t\right),
$$

where $\bar{\xi}_{k}=i_{\mathcal{N}_{0}, \mathcal{N}_{k}}(\bar{\xi})$ is the image of $\bar{\xi}$ under the iteration map induced by $\Sigma_{M_{n+1}^{-}}$.

Proof. Let $\left(\mathcal{Q}_{i} \mid i<\omega\right)$ be an enumeration of $\mathcal{F}^{+}$and $\mathcal{N}_{0}=\mathcal{N}$. Then we construct $\mathcal{N}_{k+1}$ inductively. So assume that we already constructed $\mathcal{N}_{k}$ and pseudocoiterate $\mathcal{N}_{k}$ with $\mathcal{Q}_{k}$ to some model $\mathcal{N}_{k}^{*}$ (see Lemma 3.8). By assumption $(b)$ is false, so let $\mathcal{R}$ be a counterexample witnessing this for $\mathcal{N}_{k}^{*}$ and the dlmsuitable premouse $M_{n}(x)$. That means $\mathcal{R} \in \mathcal{G}$ is the last model of a correctly guided finite stack on $\mathcal{N}_{k}^{*}$ such that $M_{n}\left(\mathcal{R} \mid \delta^{\mathcal{R}}\right)=\mathcal{P}^{M_{n}(x)}\left(\mathcal{R} \mid \delta^{\mathcal{R}}\right) \vDash \neg \varphi\left(i_{\mathcal{N}, \mathcal{R}}(\bar{\xi}), t\right)$ as $i_{\mathcal{N}, \mathcal{R}}\left\lceil H_{s}^{\mathcal{N}}=\pi_{(\mathcal{N}, s),(\mathcal{R}, s)}\right.$. But $\mathcal{R} \in \mathcal{F}^{+}$since $\mathcal{R} \in \mathcal{G}$ and it is a correct iterate of $\mathcal{Q}_{k}$. Thus we can let $\mathcal{N}_{k+1}=\mathcal{R}$.

Since $\left(\mathcal{N}_{k} \mid k<\omega\right)$ is cofinal in $\mathcal{F}^{+}$, it follows that the direct limit of $\left(\mathcal{N}_{k}, i_{\mathcal{N}_{k}, \mathcal{N}_{l}} \mid k<l<\omega\right)$ is equal to $\mathcal{M}_{\infty}^{+}$. Let $\hat{\mathcal{N}}_{k}=M_{n}\left(\mathcal{N}_{k} \mid \delta^{\mathcal{N}_{k}}\right)$ and let $\hat{i}_{\hat{\mathcal{N}}_{k}, \infty}$ : $\hat{\mathcal{N}}_{k} \rightarrow M_{n}\left(\mathcal{M}_{\infty}^{+} \mid \delta^{\mathcal{M}_{\infty}^{+}}\right)=\hat{\mathcal{M}}_{\infty}^{+}$be the corresponding extension of the direct limit map $i_{\mathcal{N}_{k}, \infty}$. Then we have for all sufficiently large $k$ that

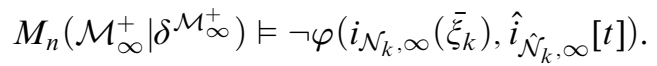

Since we assumed that $\mathcal{N}$ is strongly $s$-iterable below $\kappa$ and $\bar{\xi}<\gamma_{s}^{\mathcal{N}}$, it follows that $i_{\mathcal{N}_{k}, \infty}\left(\bar{\xi}_{k}\right)=i_{\mathcal{N}, \infty}(\bar{\xi})=\pi_{(\mathcal{N}, s), \infty}(\bar{\xi})=\xi$ as $\bar{\xi}_{k}=i_{\mathcal{N}, \mathcal{N}_{k}}(\bar{\xi})$.

Let $k<\omega$ be large enough such that $\left(\mathcal{N}_{k}, s \cup t\right) \in \mathcal{I}$ and $\hat{i}_{\hat{\mathcal{N}}_{l}, \hat{\mathcal{N}}_{l+1}}(s \cup t)=s \cup t$ for all $l \geq k$. Such a $k$ exists by a so-called bad sequence argument similar to the one in the proof of Lemma 5.8 in [12].

Consider the map

$$
\hat{\sigma}: M_{n}\left(\mathcal{M}_{\infty} \mid \delta_{\infty}\right)=\hat{\mathcal{M}}_{\infty} \rightarrow \hat{\mathcal{M}}_{\infty}^{+}=M_{n}\left(\mathcal{M}_{\infty}^{+} \mid \delta^{\mathcal{M}_{\infty}^{+}}\right)
$$

which is the canonical extension of the map $\sigma: \mathcal{M}_{\infty} \rightarrow \mathcal{M}_{\infty}^{+}$defined in the proof of Lemma 3.28. That means, for $x \in \hat{\mathcal{M}}_{\infty}$, say $x=\hat{\pi}_{\left(\mathcal{N}_{l}, r\right), \infty}(\bar{x})$ for some $\bar{x} \in H_{r}^{\hat{\mathcal{N}}_{l}}$, we may assume similar as above that $l \geq k$ is large enough such that $\left(\mathcal{N}_{l}, r\right) \in \mathcal{I}$ and $\hat{i}_{\hat{\mathcal{N}}_{j}, \hat{\mathcal{N}}_{j+1}}(r)=r$ for all $j \geq l$. Then we let $\hat{\sigma}(x)=\hat{i}_{\hat{\mathcal{N}}_{l}, \infty}(\bar{x})$. Now it follows from a generalization of the proof of [16, Claim 2] that $\hat{\sigma}=\mathrm{id}$. Moreover, we have that $\hat{\sigma}\left[t^{*}\right]=\hat{\sigma}\left(\hat{\pi}_{\left(\mathcal{N}_{k}, s \cup t\right), \infty}[t]\right)=\hat{i}_{\hat{\mathcal{N}}_{k}, \infty}[t]$. Therefore pulling back under $\hat{\sigma}$ yields that

$$
M_{n}\left(\mathcal{M}_{\infty} \mid \delta_{\infty}\right) \vDash \neg \varphi\left(\xi, t^{*}\right) .
$$

This is the desired contradiction to $(a)$. 
To show that $(b)$ implies $(a)$ we now assume that $(b)$ is true. Let $M(y)$ be the dlm-suitable premouse with $(\mathcal{N}, s) \in \mathcal{I}^{M(y)}$ given by $(b)$. As before we can assume without loss of generality that $\mathcal{N}$ is the last model of a correctly guided finite stack on $M_{n+1}^{-}$via the canonical iteration strategy $\Sigma_{M_{n+1}^{-}}$, that $\mathcal{N}$ is strongly $s$-iterable below $\kappa$ with respect to branches chosen by $\Sigma_{M_{n+1}^{-}}$, that $\max (s)$ is a uniform indiscernible, and that $\mathcal{N} \in \mathcal{G} \cap \mathcal{G}^{M(y)}$ using Lemma 3.35.

CLAIM 2. There are n-suitable premice $\mathcal{N}_{k} \in \mathcal{F}^{+}$for $k<\omega$ which are cofinal in $\mathcal{F}^{+}$ such that $\mathcal{N}_{0}=\mathcal{N}$ and for all $k<\omega$,

$$
M_{n}\left(\mathcal{N}_{k} \mid \delta^{\mathcal{N}_{k}}\right) \vDash \varphi\left(\bar{\xi}_{k}, t\right),
$$

where $\bar{\xi}_{k}=i_{\mathcal{N}_{0}, \mathcal{N}_{k}}(\bar{\xi})$ is the image of $\bar{\xi}$ under the iteration map induced by $\Sigma_{M_{n+1}^{-}}$.

Proof. By the proof of Lemma 3.35, we can pick a sequence $\left(\mathcal{Q}_{i} \mid i<\omega\right)$ of premice cofinal in $\mathcal{F}^{+}$such that $\mathcal{Q}_{i} \in \mathcal{F}^{M(y)}$ for all $i<\omega$. Let $\mathcal{N}_{0}=\mathcal{N}$ and construct $\mathcal{N}_{k+1} \in M(y)$ inductively. Assume that we already constructed $\mathcal{N}_{k}$ and let $\mathcal{M} \in$ $M(y)$ be the last model of a correctly guided finite stack on $\mathcal{N}$ witnessing that (b) is true. Simultaneously pseudo-coiterate $\mathcal{M}$ with $\mathcal{N}_{k}$ and $\mathcal{Q}_{k}$ to some premouse $\mathcal{N}_{k}^{*}$. Using genericity iterations and Lemma 3.35, there is a pseudo-iterate $\mathcal{R}$ of $\mathcal{N}_{k}^{*}$ such that $\mathcal{R} \in \mathcal{G} \cap \mathcal{G}^{M(y)}$ (see also Corollary 3.21). In particular, we have by dlm-suitability of $M(y)$ that

$$
\begin{aligned}
\mathcal{P}^{M(y)}\left(\mathcal{R} \mid \delta^{\mathcal{R}}\right) & =K^{M_{n}(x)[g]}\left(\mathcal{R} \mid \delta^{\mathcal{R}}\right)=K^{M_{n}\left(\mathcal{R} \mid \delta^{\mathcal{R}}\right)[G][g]}\left(\mathcal{R} \mid \delta^{\mathcal{R}}\right) \\
& =K^{M_{n}\left(\mathcal{R} \mid \delta^{\mathcal{R}}\right)}\left(\mathcal{R} \mid \delta^{\mathcal{R}}\right)=M_{n}\left(\mathcal{R} \mid \delta^{\mathcal{R}}\right)
\end{aligned}
$$

for some $G$ generic over $M_{n}\left(\mathcal{R} \mid \delta^{\mathcal{R}}\right)$ for the extender algebra and therefore $M_{n}\left(\mathcal{R} \mid \delta^{\mathcal{R}}\right) \vDash \varphi\left(i_{\mathcal{N}, \mathcal{R}}(\bar{\xi}), t\right)$ using $(b)$ as $i_{\mathcal{N}, \mathcal{R}}(\bar{\xi})=\pi_{(\mathcal{N}, s),(\mathcal{R}, s)}(\bar{\xi})=\pi_{(\mathcal{N}, s),(\mathcal{R}, s)}^{M(y)}(\bar{\xi})$. Moreover, $\mathcal{R}$ is the last model of a correctly guided finite stack on $\mathcal{Q}_{k}$ and thus $\mathcal{R} \in \mathcal{F}^{+}$, so we can let $\mathcal{N}_{k+1}=\mathcal{R}$.

As before we can use this claim to obtain that

$$
M_{n}\left(\mathcal{M}_{\infty} \mid \delta_{\infty}\right) \vDash \varphi\left(\xi, t^{*}\right),
$$

which proves $(a)$.

Let $\kappa_{\infty}$ be the least inaccessible cardinal above $\delta_{\infty}$ in $\hat{\mathcal{M}}_{\infty}=M_{n}\left(\mathcal{M}_{\infty} \mid \delta_{\infty}\right)$ and fix some $H$ which is $\operatorname{Col}\left(\omega,<\kappa_{\infty}\right)$-generic over $\hat{\mathcal{M}}_{\infty}$. Then Lemma 4.1 implies for example that $\hat{\mathcal{M}}_{\infty}[H]$ and $M_{n}(x)[g]$ are elementary equivalent (for formulae in the language of set theory) as for $\mathcal{R}$ as in the statement of Lemma 4.1, there is some $\operatorname{Col}\left(\omega,<\kappa^{\mathcal{R}}\right)$-generic $G$, where $\kappa^{\mathcal{R}}$ is the least inaccessible cardinal above $\delta^{\mathcal{R}}$ in $\mathcal{P}^{M(y)}\left(\mathcal{R} \mid \delta^{\mathcal{R}}\right)$, such that $\mathcal{P}^{M(y)}\left(\mathcal{R} \mid \delta^{\mathcal{R}}\right)[G]=M_{n}(x)[g]$.

We defined a direct limit system $\mathcal{F}^{M(y)}$ for all dlm-suitable $M(y)$ in $M_{n}(x)[g]$. Therefore, there is a direct limit system $\mathcal{F}^{*, M(y)}$ with the same properties for each dlm-suitable $M(y)$ in $\hat{\mathcal{M}}_{\infty}[H]$ (adapting the definition of dlm-suitable to $\hat{\mathcal{M}}_{\infty}[H]$ ). It is easy to see that Lemma 4.1 implies that for each $s \in[\text { Ord }]^{<\omega}$ there is a $y_{s}$ such that $\mathcal{M}_{\infty} \in \mathcal{G}^{M\left(y_{s}\right)}$ and $\mathcal{M}_{\infty}$ is strongly $s^{*}$-iterable in $M\left(y_{s}\right)$ in $\hat{\mathcal{M}}_{\infty}[H]$. In fact, the 
direct limit embedding $\left(\pi_{\left(\mathcal{M}_{\infty}, s^{*}\right), \infty}^{M\left(y_{s}\right)}\right)^{\mathcal{F}^{*, M\left(y_{s}\right)}}$ in the system $\mathcal{F}^{*, M\left(y_{s}\right)}$ is independent of the choice of $y_{s}$ and we can consider

$$
\begin{aligned}
\pi_{\infty} & =\bigcup\left\{\left(\pi_{\left(\mathcal{M}_{\infty}, s^{*}\right), \infty}^{M\left(y_{s}\right)}\right)^{\mathcal{F}^{*, M\left(y_{s}\right)}} \mid s \in[\mathrm{Ord}]^{<\omega} \text { and } y_{s}\right. \text { is such that } \\
& \left.\mathcal{M}_{\infty} \in \mathcal{G}^{M\left(y_{s}\right)} \text { and } \mathcal{M}_{\infty} \text { is strongly } s^{*} \text {-iterable in } M\left(y_{s}\right) \text { in } \hat{\mathcal{M}}_{\infty}[H]\right\} .
\end{aligned}
$$

Lemma 4.2. For all $\eta<\delta_{\infty}$ we have that $\pi_{\infty}(\eta)=\eta^{*}$.

Proof. This is again a consequence of Lemma 4.1. Consider the dlm-suitable premouse $M_{n}(x)$. Let $\eta=\pi_{(\mathcal{N}, s), \infty}(\bar{\eta})$ for some $(\mathcal{N}, s) \in \mathcal{I}$ and $\bar{\eta}<\gamma_{s}^{\mathcal{N}}$ and consider the formula

$$
\begin{gathered}
\varphi\left(v_{0}, v_{1}, v_{2}, v_{3}\right)=" 1 \Vdash_{\mathrm{Col}\left(\omega,<\kappa^{*}\right)} \text { for all dlm-suitable } y \text { with } v_{0} \in \mathcal{G}^{M(y)}, \\
\text { we have }\left(v_{0}, v_{1}\right) \in \mathcal{I}^{M(y)}, v_{2}<\gamma_{v_{1}}^{v_{0}, M(y)} \text {, and } \pi_{\left(v_{0}, v_{1}\right), \infty}^{M(y)}\left(v_{2}\right)=v_{3}, "
\end{gathered}
$$

where $\kappa^{*}$ refers to the least inaccessible cardinal above the least Woodin cardinal of the current model. Recall that for any dlm-suitable $y$ and $z$ witnessed by $M(y)$ and $M(z)$, for any $(\mathcal{N}, s),(\mathcal{M}, t) \in \mathcal{I}^{M(y)} \cap \mathcal{I}^{M(z)}$ with $(\mathcal{N}, s) \leq_{\mathcal{I}^{M(y)}}(\mathcal{M}, t)$ and $(\mathcal{N}, s) \leq_{\mathcal{I}^{M(z)}}(\mathcal{M}, t)$ the induced embeddings $\pi_{(\mathcal{N}, s),(\mathcal{M}, t)}^{M(y)}$ and $\pi_{(\mathcal{N}, s),(\mathcal{M}, t)}^{M(z)}$ agree. Hence, in $M_{n}(x)[g]$, we have for every $\mathcal{R} \in \mathcal{G}$ which is the last model of a correctly guided finite stack on $\mathcal{N}$ that for all dlm-suitable $y$ such that $\mathcal{R} \in \mathcal{G}^{M(y)}$, in fact $(\mathcal{R}, s) \in \mathcal{I}^{M(y)}, \pi_{(\mathcal{N}, s),(\mathcal{R}, s)}(\bar{\eta})<\gamma_{s}^{\mathcal{R}, M(y)}$, and $\pi_{(\mathcal{R}, s), \infty}^{M(y)}\left(\pi_{(\mathcal{N}, s),(\mathcal{R}, s)}(\bar{\eta})\right)=\eta$. Therefore, $\mathcal{P}^{M(y)}\left(\mathcal{R} \mid \delta^{\mathcal{R}}\right) \vDash \varphi\left(\mathcal{R}, s, \pi_{(\mathcal{N}, s),(\mathcal{R}, s)}(\bar{\eta}), \eta\right)$. So Lemma 4.1 yields that $\hat{\mathcal{M}}_{\infty} \vDash \varphi\left(\mathcal{M}_{\infty}, s^{*}, \eta, \eta^{*}\right)$. So in $\hat{\mathcal{M}}_{\infty}[H]$, for all dlm-suitable $y$ with $\mathcal{M}_{\infty} \in \mathcal{G}^{M(y)}$, we have $\left(\mathcal{M}_{\infty}, s^{*}\right) \in \mathcal{I}^{M(y)}$ and $\left(\pi_{\left(\mathcal{M}_{\infty}, s^{*}\right), \infty}^{M(y)}\right)^{\mathcal{F}^{*}, M(y)}(\eta)=\eta^{*}$, as desired.

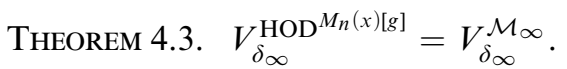

Proof. By the internal definition of $\mathcal{M}_{\infty}$ from Lemma 3.35 we have that $V_{\delta_{\infty}}^{\mathcal{M}_{\infty}} \subseteq$

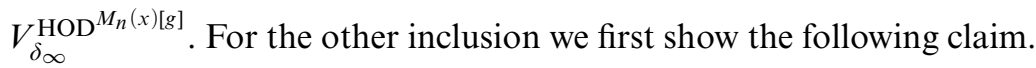

Claim 1. $\pi_{\infty}\left\lceil\alpha \in \hat{\mathcal{M}}_{\infty}\right.$ for all $\alpha<\delta_{\infty}$.

Proof. As $\alpha<\delta_{\infty}$, there exists an $s \in[\text { Ord }]^{<\omega}$ and a dlm-suitable $y_{s}$ such that, in $\hat{\mathcal{M}}_{\infty}[H], \mathcal{M}_{\infty} \in \mathcal{G}^{M(y)}$ and $\alpha<\gamma_{s^{*}}^{\mathcal{M}_{\infty}, M\left(y_{s}\right)}$. We have by definition that $\pi_{\infty}$ । $\alpha=\left(\pi_{\left(\mathcal{M}_{\infty}, s^{*}\right), \infty}^{M\left(y_{s}\right)}\right)^{\mathcal{F}^{*}, M\left(y_{s}\right)}\left\lceil\alpha\right.$. As $\left(\pi_{\left(\mathcal{M}_{\infty}, s^{*}\right), \infty}^{M\left(y_{s}\right)}\right)^{\mathcal{F}^{*}, M\left(y_{s}\right)} \uparrow \alpha$ does not depend on the choice of $y_{s}$, this implies $\pi_{\infty}\left\lceil\alpha \in \operatorname{HOD}_{\mathcal{M}_{\infty}}^{\hat{\mathcal{M}}_{\infty}[H]}\right.$ and thus $\left.\pi_{\infty}\right\rceil \alpha \in \hat{\mathcal{M}}_{\infty}$ by homogeneity of the forcing $\mathbb{P}=\operatorname{Col}\left(\omega,<\kappa_{\infty}\right)$.

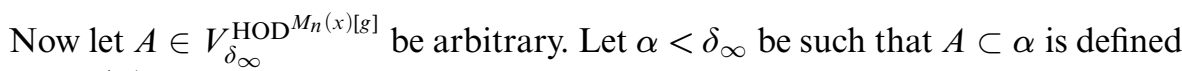
over $M_{n}(x)[g]$ by a formula $\varphi$ with ordinal parameters from $t \in[\mathrm{Ord}]^{<\omega}$ and let $\beta<\alpha$ be arbitrary. That means $\beta \in A$ iff $M_{n}(x)[g] \vDash \varphi(\beta, t)$. Lemma 4.1 yields that this is the case iff $\hat{\mathcal{M}}_{\infty}[H] \vDash \varphi\left(\beta^{*}, t^{*}\right)$. Since $\beta<\alpha<\delta_{\infty}$, we have that $\beta^{*}=\pi_{\infty}(\beta)$ by Lemma 4.2. Moreover, we have by Claim 1 that $\pi_{\infty}\left\lceil\alpha \in \hat{\mathcal{M}}_{\infty}\right.$. Therefore, it 
follows by homogeneity of the forcing $\mathbb{P}=\operatorname{Col}\left(\omega,<\kappa_{\infty}\right)$ that $A \in \hat{\mathcal{M}}_{\infty}$ since $t^{*}$ is a fixed parameter in $\hat{\mathcal{M}}_{\infty}$. Thus $A \in V_{\delta_{\infty}}^{\mathcal{M}_{\infty}}$, as desired.

§5. The full HOD in $M_{n}(x)[g]$. To compute the full model $\operatorname{HOD}^{M_{n}(x)[g]}$, i.e., prove Theorem 1.1, we first show the following lemma. Recall that $\omega_{2}^{M_{n}(x)[g]}=\delta_{\infty}$.

Lemma 5.1. $\operatorname{HOD}^{M_{n}(x)[g]}=M_{n}(A)$ for some set $A \subseteq \omega_{2}^{M_{n}(x)[g]}$ with $A \in$ $\operatorname{HOD}^{M_{n}(x)[g]}$.

Proof. Let $\mathbb{V}$ denote the Vopěnka algebra in $M_{n}(x)[g]$ for making a real generic over $\operatorname{HOD}^{M_{n}(x)[g]}$. By Vopěnka's theorem (see for example Theorem 15.46 in [4] or Theorem 9.0.1 in [6]) there is a $\mathbb{V}$-generic $G_{x}$ over $\operatorname{HOD}^{M_{n}(x)[g]}$ such that $x \in$ $\operatorname{HOD}^{M_{n}(x)[g]}\left[G_{x}\right]$ and in fact $\operatorname{HOD}^{M_{n}(x)[g]}\left[G_{x}\right]=\operatorname{HOD}_{x}^{M_{n}(x)[g]}$.

ClaIm 1. There is some $\tilde{\mathbb{V}} \in \mathrm{HOD}^{M_{n}(x)[g]}$ which is isomorphic to $\mathbb{V}$ and a subset of $\omega_{2}^{M_{n}(x)[g]}$ ? $^{7}$

Proof. Work in $M_{n}(x)[g]$. Each real, i.e., element of $\mathcal{P}(\omega)$, can be coded by a countable ordinal and each set of reals can be coded by an ordinal $<\omega_{2}$. Forcing with the Vopěnka algebra $\mathbb{V}$ is $\omega_{2}$-c.c. in $M_{n}(x)[g]$ as otherwise there would be an $\omega_{2}$ sequence of pairwise distinct non-empty sets of reals, contradicting $\mathrm{CH}$. The Vopěnka algebra is in $\operatorname{HOD}^{M_{n}(x)[g]}$ and when considering $\operatorname{HOD}^{M_{n}(x)[g]}\left[G_{x}\right]$ cardinals $\geq\left(\kappa^{+}\right)^{M_{n}(x)}$ are preserved. Since $\left(\kappa^{+\omega}\right)^{M_{n}(x)}$ is below the least measurable cardinal of $M_{n}(x), M_{n}(x) \mid\left(\kappa^{+\omega}\right)^{M_{n}(x)}$ can be written as the $L p^{n}$-stack of height $\left(\kappa^{+\omega}\right)^{M_{n}(x)}$ above $x$ and is therefore by the argument in Lemma 3.18 an element of $\operatorname{HOD}_{x}^{M_{n}(x)[g]}=\operatorname{HOD}^{M_{n}(x)[g]}\left[G_{x}\right]$. But the Vopěnka algebra is a subset of some ordinal $\alpha<\left(\kappa^{++}\right)^{M_{n}(x)}=\left(\kappa^{++}\right)^{\mathrm{HOD}^{M_{n}(x)[g]}\left[G_{x}\right]}=\left(\kappa^{++}\right)^{\mathrm{HOD}^{M_{n}(x)[g]}}$, so there is some $\tilde{\mathbb{V}} \in \operatorname{HOD}^{M_{n}(x)[g]}$ which is isomorphic to $\mathbb{V}$ and a subset of $\left(\kappa^{+}\right)^{M_{n}(x)}$.

For the rest of this proof we write $\mathbb{V}$ for the $\tilde{\mathbb{V}}$ from the previous claim and show that $M_{n}(\mathbb{V})=\operatorname{HOD}^{M_{n}(x)[g]}$.

Claim 2. $G_{x}$ is $\mathbb{V}$-generic over $M_{n}(\mathbb{V})$.

Proof. The dense sets in question are elements of $\mathcal{P}(\mathbb{V})^{M_{n}(\mathbb{V})}$ and hence elements of $L p^{n}(\mathbb{V})=\bigcup\left\{N \mid N\right.$ is a countable $\mathbb{V}$-premouse with $\rho_{\omega}(N)=\mathbb{V}$ which is $n$-small, sound, and $\left(\omega, \omega_{1}, \omega_{1}\right)$-iterable $\}$. As $\mathbb{V} \in \operatorname{HOD}^{M_{n}(x)[g]}$, Lemma 3.18 yields that $L p^{n}(\mathbb{V}) \in \operatorname{HOD}^{M_{n}(x)[g]}$, which implies the claim.

Let $\lambda$ be the least inaccessible of $M_{n}(x)$ above $\kappa$.

Claim 3. $V_{\lambda}^{M_{n}(x)}=V_{\lambda}^{M_{n}(\mathbb{V})\left[G_{x}\right]}$ and $\lambda$ is a cardinal in $M_{n}(\mathbb{V})\left[G_{x}\right]$.

Proof. Write $\kappa_{0}^{M_{n}(x)}$ and $\kappa_{0}^{M_{n}(\mathbb{V})}$ for the least measurable cardinal of $M_{n}(x)$ and $M_{n}(\mathbb{V})$ respectively. $M_{n}(\mathbb{V})$ and $M_{n}(\mathbb{V})\left[G_{x}\right]$ have the same least measurable cardinal. The proof of Lemma 3.18 shows that $L p^{n}(z) \in M_{n}(\mathbb{V})\left[G_{x}\right]$ for any $z \in V_{\delta_{0}}^{M_{n}(\mathbb{V})\left[G_{x}\right]}$,

\footnotetext{
${ }^{7}$ We would like to thank the anonymous referee for pointing out that this was overlooked in an earlier version of this article and for suggesting the argument we provide here.
} 
where $\delta_{0}$ denotes the least Woodin cardinal in $M_{n}(\mathbb{V})\left[G_{x}\right]$. As $M_{n}(x) \mid \kappa_{0}^{M_{n}(x)}$ is equal to the $L p^{n}$-stack of height $\kappa_{0}^{M_{n}(x)}$ over $x$, it follows that

$$
M_{n}(x) \mid \kappa_{0}^{M_{n}(x)} \subseteq M_{n}(\mathbb{V})\left[G_{x}\right] .
$$

Analogously, $M_{n}(\mathbb{V}) \mid \kappa_{0}^{M_{n}(\mathbb{V})} \subseteq M_{n}(x)$ and in fact

$$
M_{n}(\mathbb{V})\left[G_{x}\right] \mid \kappa_{0}^{M_{n}(\mathbb{V})} \subseteq M_{n}(x) .
$$

We are left with showing that $\lambda<\kappa_{0}^{M_{n}(\mathbb{V})}$ (as $\lambda<\kappa_{0}^{M_{n}(x)}$ is obvious). So suppose toward a contradiction that $\lambda \geq \kappa_{0}^{M_{n}(\mathbb{V})}$. Then

$$
V_{\kappa_{0}^{M_{n}(\mathbb{V})}}^{M_{n}(\mathbb{V})\left[G_{x}\right]}=V_{\kappa_{0}^{M_{n}(\mathbb{V})}}^{M_{n}(x)}
$$

Therefore, $\kappa_{0}^{M_{n}(\mathbb{V})}$ is not only a limit of inaccessible cardinals in $M_{n}(\mathbb{V})\left[G_{x}\right]$ but also in $M_{n}(x)$, contradicting our assumption that $\lambda \geq \kappa_{0}^{M_{n}(\mathbb{V})}$.

CLAIM 4. $V_{\lambda}^{M_{n}(\mathbb{V})} \subseteq \operatorname{HOD}^{M_{n}(x)[g]}$.

Proof. This follows from the proof of Lemma 3.18 as $M_{n}(\mathbb{V}) \mid \lambda$ can be obtained as the $L p^{n}$-stack of height $\lambda$ over $\mathbb{V}$ and $\mathbb{V} \in \operatorname{HOD}^{M_{n}(x)[g]}$.

Now we can show that the lemma holds below $\lambda$.

Claim 5. $V_{\lambda}^{M_{n}(\mathbb{V})}=V_{\lambda}^{\mathrm{HOD}^{M_{n}(x)[g]}}$.

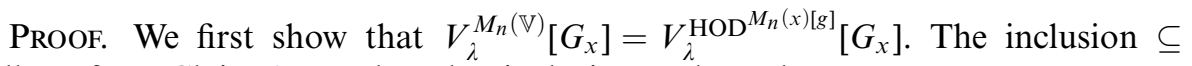
follows from Claim 4. For the other inclusion we have that

$$
\operatorname{HOD}^{M_{n}(x)[g]}\left[G_{x}\right]=\operatorname{HOD}_{x}^{M_{n}(x)[g]} \subseteq \operatorname{HOD}_{x}^{M_{n}(x)} \subseteq M_{n}(x),
$$

using the homogeneity and ordinal definability of the forcing $\operatorname{Col}(\omega,<\kappa)$. Therefore by Claim 3

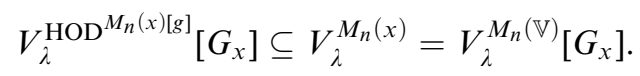

Finally, we argue that the equality $V_{\lambda}^{M_{n}(\mathbb{V})}\left[G_{x}\right]=V_{\lambda}^{\operatorname{HOD}^{M_{n}(x)[g]}}\left[G_{x}\right]$ also holds true

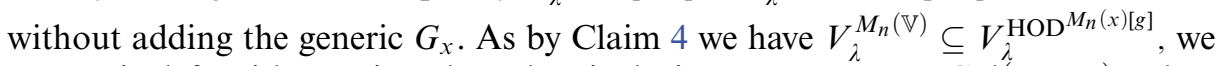
are again left with proving the other inclusion. Let $\mathbb{P} \stackrel{\lambda}{=} \mathbb{V} \times \operatorname{Col}(\omega,<\kappa)$. Then

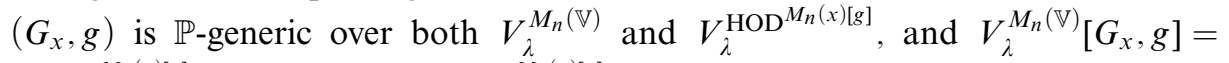
$V_{\lambda}^{\mathrm{HOD}^{M_{n}(x)[g]}}\left[G_{x}, g\right]$. Let $a \in V_{\lambda}^{\mathrm{HOD}^{M_{n}(x)[g]}}$ be a set of ordinals. Then there is a $\mathbb{P}$-name $\sigma \in V_{\lambda}^{M_{n}(\mathbb{V})}$ such that $\sigma_{\left(G_{x}, g\right)}=a$. This is forced over $V_{\lambda}^{\mathrm{HOD}^{M_{n}(x)[g]}}$, i.e., there is a $p \in \mathbb{P}$ such that $V_{\lambda}^{\mathrm{HOD}^{M_{n}(x)[g]} \vDash " ~} p \Vdash \sigma=\check{a}$." Thus $V_{\lambda}^{M_{n}(\mathbb{V})}$ can compute the elements of $a$ using the forcing relation for $\mathbb{P}$ below $p$. Hence $a \in V_{\lambda}^{M_{n}(\mathbb{V})}$, as desired.

Now we are able to extend Claim 3 to the full models.

Claim 6. $M_{n}(\mathbb{V})\left[G_{x}\right]=M_{n}(x)$.

Proof. Consider $M_{n}(x)[g]$ as a $V_{\lambda}^{M_{n}(x)[g]}$-premouse and note that it equals $M_{n}\left(V_{\lambda}^{M_{n}(x)[g]}\right)$. We use $\mathcal{P}^{M_{n}(x)[g]}\left(M_{n}(\mathbb{V}) \mid \lambda\right)$ to denote the result of a $\mathcal{P}$-construction 
in the sense of [15] above $M_{n}(\mathbb{V}) \mid \lambda$ inside the $V_{\lambda}^{M_{n}(x)[g]}$-premouse $M_{n}(x)[g]$. By Claim 3, $V_{\lambda}^{M_{n}(\mathbb{V})}\left[G_{x}\right]=V_{\lambda}^{M_{n}(x)}$, so $V_{\lambda}^{M_{n}(\mathbb{V})}\left[G_{x}\right][g]=V_{\lambda}^{M_{n}(x)[g]}$ and this $\mathcal{P}$ construction is well-defined. Moreover, the following argument shows that the construction never projects across $\lambda$.

Assume toward a contradiction that there is a level $\mathcal{P}$ of the $\mathcal{P}$-construction above $M_{n}(\mathbb{V}) \mid \lambda$ inside $M_{n}(x)[g]$ such that $\rho_{\omega}(\mathcal{P})=\rho<\lambda$. That means there is an $r \Sigma_{k+1}(\mathcal{P})$-definable set $a \subseteq \rho$ for some $k<\omega$ such that $a \notin \mathcal{P}$. As by the proof of Claim $4, M_{n}(\mathbb{V}) \mid \lambda \in \operatorname{HOD}^{M_{n}(x)[g]}$ it follows by definability of the $\mathcal{P}$-construction and of the extender sequence of $M_{n}\left(V_{\lambda}^{M_{n}(x)[g]}\right.$ ) (see Lemma 1.1 in [14] due to J. Steel) that $\mathcal{P} \in \operatorname{HOD}^{M_{n}(x)[g]}$. This means that in particular $a \in \operatorname{HOD}^{M_{n}(x)[g]}$. But

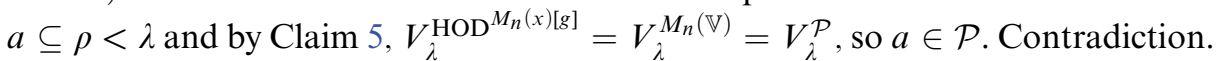

Now it follows by construction (see [15]) that

$$
\mathcal{P}^{M_{n}(x)[g]}\left(M_{n}(\mathbb{V}) \mid \lambda\right)\left[G_{x}\right][g]=M_{n}(x)[g] .
$$

But this yields that $\mathcal{P}^{M_{n}(x)[g]}\left(M_{n}(\mathbb{V}) \mid \lambda\right)\left[G_{x}\right]=M_{n}(x)$, without adding the generic $g$, by an argument as the one at the end of the previous claim. Moreover, $\mathcal{P}^{M_{n}(x)[g]}\left(M_{n}(\mathbb{V}) \mid \lambda\right)=M_{n}(\mathbb{V})$ and thus $M_{n}(\mathbb{V})\left[G_{x}\right]=M_{n}(x)$, as desired.

This argument also shows the following claim.

Claim 7. $M_{n}(\mathbb{V}) \subseteq \mathrm{HOD}^{M_{n}(x)[g]}$.

Now, the next claim follows from the first half of the proof of Claim 5.

Claim 8. $M_{n}(\mathbb{V})\left[G_{x}\right]=\operatorname{HOD}^{M_{n}(x)[g]}\left[G_{x}\right]$.

Finally, the statement of Claim 8 also holds true without adding the generic $G_{x}$ by the argument at the end of the proof of Claim 5. Hence $M_{n}(\mathbb{V})=\operatorname{HOD}^{M_{n}(x)[g]}$, as desired.

Corollary 5.2. Let $F(s)=s^{*}$ for $s \in[\mathrm{Ord}]^{<\omega}$. Then

$$
\operatorname{HOD}^{M_{n}(x)[g]}=M_{n}\left(\mathcal{M}_{\infty} \mid \delta_{\infty}, F\left\lceil\delta_{\infty}\right)\right.
$$

Proof. Note that $\mathcal{M}_{\infty} \mid \delta_{\infty}$ and $F\left\lceil\delta_{\infty}\right.$ are elements of $\operatorname{HOD}^{M_{n}(x)[g]}$ by construction. Let $\eta=\sup F " \delta_{\infty}$ and let $\gamma$ be the least inaccessible cardinal of $M_{n}\left(\mathcal{M}_{\infty} \mid \delta_{\infty}, F\left\lceil\delta_{\infty}\right)\right.$ above $\eta$. Let $A \subseteq \omega_{2}^{M_{n}(x)[g]}$ be as in the statement of Lemma 5.1, i.e., such that $\operatorname{HOD}^{M_{n}(x)[g]}=M_{n}(A)$. Moreover, let $\varphi$ be a formula defining $A$, i.e., $\xi \in A$ iff $M_{n}(x)[g] \vDash \varphi(\xi)$. Then, as $F(\xi)=\pi_{\infty}(\xi)$ for $\xi<\delta_{\infty}$ by Lemma 4.2,

$$
\begin{array}{rl}
\xi \in A \text { iff } M_{n}\left(\mathcal{M}_{\infty} \mid \delta_{\infty}\right) \vDash " & 1 \Vdash^{\mathbb{P}} \varphi\left(\pi_{\infty}(\xi)\right) \text {, where } \pi_{\infty} \text { is the direct limit } \\
& \text { embedding from the systems on } \mathcal{M}_{\infty} "
\end{array}
$$

for $\mathbb{P}=\operatorname{Col}\left(\omega,<\kappa_{\infty}\right)$. Consider $L[E]\left(\mathcal{M}_{\infty} \mid \delta_{\infty}\right)^{M_{n}\left(\mathcal{M}_{\infty}\left|\delta_{\infty}, F\right| \delta_{\infty}\right)}$, the result of a fully backgrounded extender construction in the sense of [7] inside $M_{n}\left(\mathcal{M}_{\infty} \mid \delta_{\infty}, F \uparrow\right.$ $\left.\delta_{\infty}\right)$ above $\mathcal{M}_{\infty} \mid \delta_{\infty}$. The premice $M_{n}\left(\mathcal{M}_{\infty} \mid \delta_{\infty}\right)$ and $L[E]\left(\mathcal{M}_{\infty} \mid \delta_{\infty}\right)^{M_{n}\left(\mathcal{M}_{\infty}\left|\delta_{\infty}, F\right| \delta_{\infty}\right)}$ successfully compare to a common proper class premouse without drops on the main 
branches. Since the iterations take place above $\delta_{\infty}, \xi<\delta_{\infty}$ is not moved and we have by elementarity

$$
\begin{array}{r}
\xi \in A \text { iff } L[E]\left(\mathcal{M}_{\infty} \mid \delta_{\infty}\right)^{M_{n}\left(\mathcal{M}_{\infty}\left|\delta_{\infty}, F\right| \delta_{\infty}\right)} \vDash " 1 \Vdash^{\mathbb{P}} \varphi\left(\pi_{\infty}(\xi)\right), \text { where } \pi_{\infty} \\
\text { is the direct limit embedding from the systems on } \mathcal{M}_{\infty} . "
\end{array}
$$

Therefore it follows that $A \in M_{n}\left(\mathcal{M}_{\infty} \mid \delta_{\infty}, F\left\lceil\delta_{\infty}\right)\right.$.

By the same argument as in the proof of Claim 3 in the proof of Lemma 5.1 we now obtain that $\gamma$ is also the least inaccessible cardinal above $\eta$ of $M_{n}(A)$ and the universes of $M_{n}\left(\mathcal{M}_{\infty} \mid \delta_{\infty}, F\left\lceil\delta_{\infty}\right)\right.$ and $M_{n}(A)$ agree up to $\gamma$. In particular, we can rearrange $M_{n}\left(\mathcal{M}_{\infty} \mid \delta_{\infty}, F\left\lceil\delta_{\infty}\right)\right.$ and $M_{n}(A)$ as $V_{\gamma}^{M_{n}\left(\mathcal{M}_{\infty}\left|\delta_{\infty}, F\right| \delta_{\infty}\right)}$-premice. As such it follows that the following equalities for classes (not structures) hold:

$$
\begin{aligned}
M_{n}\left(\mathcal{M}_{\infty} \mid \delta_{\infty}, F\left\lceil\delta_{\infty}\right)\right. & =M_{n}\left(V_{\gamma}^{M_{n}\left(\mathcal{M}_{\infty}\left|\delta_{\infty}, F\right| \delta\right)}\right) \\
& =M_{n}(A)=\operatorname{HOD}^{M_{n}(x)[g]} .
\end{aligned}
$$

The following corollary follows immediately from Lemma 4.2 and Corollary 5.2.

COROLlary 5.3. $\operatorname{HOD}^{M_{n}(x)[g]}=M_{n}\left(\mathcal{M}_{\infty} \mid \delta_{\infty}, \pi_{\infty}\left\lceil\delta_{\infty}\right)\right.$.

We now consider the iteration strategy for $\mathcal{M}_{\infty}$. Let $\Lambda$ be the restriction of $\Sigma_{M_{n+1}^{-}}$ to correctly guided finite stacks $\overrightarrow{\mathcal{T}}$ on $\mathcal{M}_{\infty} \mid \delta_{\infty}$ such that $\overrightarrow{\mathcal{T}} \in \hat{\mathcal{M}}_{\infty} \mid \kappa_{\infty}$, where $\kappa_{\infty}$ is the least inaccessible cardinal in $\hat{\mathcal{M}}_{\infty}$ above $\delta_{\infty}$.

LeMmA 5.4. $\Lambda \in \operatorname{HOD}^{M_{n}(x)[g]}$.

Proof. Let $\mathcal{T}$ be a maximal tree on $\mathcal{M}_{\infty} \mid \delta_{\infty}$ with $\mathcal{T} \in \hat{\mathcal{M}}_{\infty} \mid \kappa_{\infty}$. Moreover, let $b=\Lambda(\mathcal{T})$. Let $\mathcal{R}=\mathcal{M}_{b}^{\mathcal{T}}$ be the last model of $\mathcal{T}^{\frown} b$. Then $\mathcal{R} \in \operatorname{HOD}^{M_{n}(x)[g]}$. Moreover, let $\delta_{\infty}^{\mathcal{F}^{*}}$ be the least Woodin cardinal in $\mathcal{M}_{\infty}^{\mathcal{F}^{*}}$, the direct limit of the system $\mathcal{F}^{*, M(y)}$ for some/all dlm-suitable $M(y)$ in $\hat{\mathcal{M}}_{\infty}[H]$. Then $\mathcal{M}_{\infty}^{\mathcal{F}^{*}} \mid \delta_{\infty}^{\mathcal{F}^{*}}$ is an iterate of $\mathcal{R}$. As $\pi_{\infty}\left\lceil\delta_{\infty} \in \operatorname{HOD}^{M_{n}(x)[g]}\right.$, we can identify $b$ inside $M_{n}(x)[g]$ as the unique branch through $\mathcal{T}$ which is $\left(\pi_{\infty}\left\lceil\delta_{\infty}\right)\right.$-realizable, i.e., such that there is an elementary embedding $\sigma: \mathcal{M}_{b}^{\mathcal{T}} \rightarrow \mathcal{M}_{\infty}^{\mathcal{F}^{*}} \mid \delta_{\infty}^{\mathcal{F}^{*}}$ with $\pi_{\infty}\left\lceil\delta_{\infty}=\sigma \circ i_{b}^{\mathcal{T}}\right.$.

The same argument applies to pseudo-normal iterates $\mathcal{N}$ of $\mathcal{M}_{\infty}$ with $\mathcal{N} \mid \delta^{\mathcal{N}} \in$ $\hat{\mathcal{M}}_{\infty} \mid \kappa_{\infty}$ and maximal iteration trees $\mathcal{T}$ on $\mathcal{N} \mid \delta^{\mathcal{N}}$ such that $\mathcal{T} \in \hat{\mathcal{M}}_{\infty} \mid \kappa_{\infty}$, hence $\Lambda \in \operatorname{HOD}^{M_{n}(x)[g]}$.

Similarly to Lemma 3.47 in [23] we finally need a method of Boolean-valued comparison. As the proof is analogous we omit it.

Lemma 5.5. Let $H$ be $\operatorname{Col}\left(\omega,<\kappa_{\infty}\right)$-generic over $\hat{\mathcal{M}}_{\infty}$, and let $\mathcal{Q}$ be such that $\hat{\mathcal{M}}_{\infty}[H] \vDash$ "Q $\mathcal{Q}$ is countable and $n$-suitable." Then there is an $\mathcal{R}$ such that

(1) $\mathcal{R}$ is a pseudo-normal iterate of $\mathcal{Q}$,

(2) $\mathcal{R}$ is a $\Sigma_{M_{n+1}^{-}}$-iterate of $\mathcal{M}_{\infty}$, and

(3) $\mathcal{R} \in \hat{\mathcal{M}}_{\infty}$.

Finally, we can finish the proof of Theorem 1.1.

THEOREM 5.6.

$$
\operatorname{HOD}^{M_{n}(x)[g]}=M_{n}\left(\mathcal{M}_{\infty} \mid \delta_{\infty}, \pi_{\infty}\left\lceil\delta_{\infty}\right)=M_{n}\left(\hat{\mathcal{M}}_{\infty} \mid \kappa_{\infty}, \Lambda\right)\right.
$$


Proof. $\operatorname{HOD}^{M_{n}(x)[g]}=M_{n}\left(\mathcal{M}_{\infty} \mid \delta_{\infty}, \pi_{\infty}\left\lceil\delta_{\infty}\right)\right.$ is Corollary 5.3. Moreover, $\quad M_{n}\left(\mathcal{M}_{\infty} \mid \delta_{\infty}, \pi_{\infty}\left\lceil\delta_{\infty}\right)=M_{n}\left(\hat{\mathcal{M}}_{\infty} \mid \kappa_{\infty}, \Lambda\right)\right.$ follows from Lemma 5.5 as follows. First, $\Lambda \in M_{n}\left(\mathcal{M}_{\infty} \mid \delta_{\infty}, \pi_{\infty}\left\lceil\delta_{\infty}\right)=\operatorname{HOD}^{M_{n}(x)[g]}\right.$ and $\hat{\mathcal{M}}_{\infty} \mid \kappa_{\infty} \in$ $M_{n}\left(\mathcal{M}_{\infty} \mid \delta_{\infty}, \pi_{\infty}\left\lceil\delta_{\infty}\right)\right.$ by considering the $L p^{n}$-stack on $\mathcal{M}_{\infty} \mid \delta_{\infty}$. The direct limit of $\mathcal{F}^{*, M(y)}$ for some $M(y)$ is the same as the direct limit of all $\Lambda$ iterates of $\mathcal{M}_{\infty}$ which are an element of $\hat{\mathcal{M}}_{\infty} \mid \kappa_{\infty}$ via the comparison maps. Moreover, we have that $\pi_{\infty}$ is the canonical direct limit map of this system and therefore definable from $\hat{\mathcal{M}}_{\infty} \mid \kappa_{\infty}$ and $\Lambda$. So $\pi_{\infty}\left\lceil\delta_{\infty} \in M_{n}\left(\hat{\mathcal{M}}_{\infty} \mid \kappa_{\infty}, \Lambda\right)\right.$. Now, $M_{n}\left(\mathcal{M}_{\infty}\left|\delta_{\infty}, \pi_{\infty}\right| \delta_{\infty}\right)=M_{n}\left(\hat{\mathcal{M}}_{\infty} \mid \kappa_{\infty}, \Lambda\right)$ follows analogous to the proof of Corollary 5.2.

Note that Theorem 4.3 and Lemma 5.1 together imply Corollary 1.2, i.e., that the $\mathrm{GCH}$ holds in $\mathrm{HOD}^{M_{n}(x)[g]}$. Finally, most of the arguments we gave in this and the previous sections generalize with only small changes to more arbitrary canonical self-iterable inner models, e.g., $M_{\omega}, M_{\omega+42}$. We leave the details to the reader.

Acknowledgments. The authors thank Farmer Schlutzenberg for the helpful discussions during the 4th Münster conference on inner model theory in the summer of 2017. Finally, the authors thank the referee for carefully reading the paper and making several helpful comments and suggestions.

Part of this work was done whilst the authors were visiting fellows at the Isaac Newton Institute for Mathematical Sciences in the programme Mathematical, Foundational and Computational Aspects of the Higher Infinite (HIF) funded by EPSRC grant EP/K032208/1. The first author, formerly known as Sandra Uhlenbrock, was partially supported by FWF grant number P 28157 and in addition gratefully acknowledges funding from L'ORÉAL Austria, in collaboration with the Austrian UNESCO Commission and in cooperation with the Austrian Academy of Sciences-Fellowship Determinacy and Large Cardinals. The second author was partially supported by the NSF Career Award DMS-1352034.

\section{REFERENCES}

[1] H. S. BECKER, Thin collections of sets of projective ordinals and analogs of L. Annals of Mathematical Logic, vol. 19 (1980), no. 3, pp. 205-241.

[2] I. FARAH. The extender algebra and $\Sigma_{1}^{2}$-absoluteness, Large Cardinals, Determinacy, and Other Topics: The Cabal Seminar, vol. IV (A. S. Kechris, B. Löwe, and J. R. Steel, editors), Cambridge University Press, Cambridge, 2021.

[3] G. Fuchs, J. D. Hamkins, and J. Reitz, Set-theoretic geology. Annals of Pure and Applied Logic, vol. 166 (2015), no. 4, pp. 464-501.

[4] T. J. JeCh, Set Theory, Springer Monographs in Mathematics, Springer, Berlin, 2003.

[5] P. Koellner and W. H. Woodin, Large cardinals from determinacy, Handbook of Set Theory (M. Foreman and A. Kanamori, editors), Springer, Berlin, 2010.

[6] P. B. LaRson, Extensions of the Axiom of Determinacy, 2017. Preprint available at http://www.users.miamioh.edu/larsonpb/adplusbook_public.pdf.

[7] W. J. Mitchell and J. R. Steel, Fine Structure and Iteration Trees, Lecture Notes in Logic, Springer-Verlag, Berlin, New York, 1994.

[8] S. Müller, R. SChindler, and W. H. Woodin, Mice with finitely many Woodin cardinals from optimal determinacy hypotheses. Journal of Mathematical Logic, vol. 20 (2020), Article No. 1950013.

[9] I. Neeman, Optimal proofs of determinacy. Bulletin of Symbolic Logic, vol. 1 (1995), no. 3, pp. 327-339. 
[10] - Optimal proofs of determinacy II. Journal of Mathematical Logic, vol. 2 (2002), no. 2, pp. 227-258.

[11] G. Sargsyan, A Tale of Hybrid Mice, Ph.D. thesis, University of California, Berkeley, 2009.

[12] - On the prewellorderings associated with directed systems of mice, this JourNaL, vol. 78 (2013), no. 3, pp. 735-763.

[13] G. Sargsyan and R. Schindler, Varsovian models I, this Journal, vol. 83 (2018), no. 2, pp. $496-528$.

[14] R. SCHINDLER, Core models in the presence of Woodin cardinals, this JouRNAL, vol. 71 (2006), no. 4, pp. $1145-1154$.

[15] R. SchindLer and J. R. Steel, The self-iterability of $L$ [E], this Journal, vol. 74 (2009), no. 3 , pp. 751-779.

[16] F. Schlutzenberg. " $\Theta$ is Woodin in HOD” directly from mice, 2016. Available at https://www.math.uci.edu/ mzeman/CMI/talk-schlutzenberg-4.pdf.

[17] F. Schlutzenberg and N. Trang, Scales in hybrid mice over $\mathbb{R}$, preprint, 2016. Available at https://arxiv.org/abs/1210.7258.

[18] J. R. SteEL, Inner models with many Woodin cardinals. Annals of Pure and Applied Logic, vol. 65 (1993), pp. 185-209.

[19],$H O D^{L(\mathbb{R})}$ is a core model below $\Theta$. The Bulletin of Symbolic Logic, vol. 1 (1995), no. 1 , pp. $75-84$.

[20] - Derived models associated to mice, Computational Prospects of Infinity - Part I (C.-T. Chong, editor), Lecture Notes Series, vol. 14, World Scientific, Singapore, 2008.

[21] - An outline of inner model theory, Handbook of Set Theory (M. Foreman and A. Kanamori, editors), Springer, Berlin, 2010.

[22] - Normalizing iteration trees and comparing iteration strategies, preprint, 2016. Available at https://math.berkeley.edu/ steel/papers.

[23] J. R. STEEL and W. H. WoOdIn, HOD as a core model, Ordinal Definability and Recursion Theory, The Cabal Seminar, vol. III (A. S. Kechris, B. Löwe, and J. R. Steel, editors), Cambridge University Press, Cambridge, 2016.

[24] T. Usuba, The downward directed grounds hypothesis and very large cardinals. Journal of Mathematical Logic, vol. 17 (2017), no. 02, Article No. 1750009.

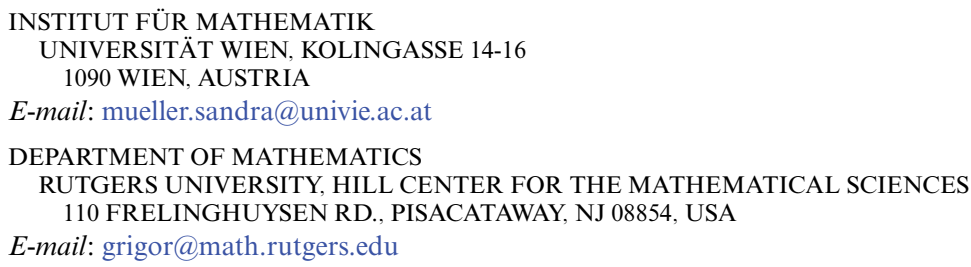

E-mail: mueller.sandra@univie.ac.at

DEPARTMENT OF MATHEMATICS

RUTGERS UNIVERSITY, HILL CENTER FOR THE MATHEMATICAL SCIENCES

110 FRELINGHUYSEN RD., PISACATAWAY, NJ 08854, USA

E-mail: grigor@math.rutgers.edu 